\title{
Integrating Children with Psychiatric Disorders in the Classroom: A Systematic Review
}

\author{
Giulia Cossu ${ }^{1, *}$, Elisa Cantone ${ }^{1}$, Mirra Pintus ${ }^{1}$, Michela Cadoni ${ }^{1}$, Anna Pisano ${ }^{1}$, Roy Otten ${ }^{2}$, \\ Rowella Kuijpers ${ }^{2}$, Elisa Pintus ${ }^{1}$, Federica Sancassiani ${ }^{1}$, Maria Francesca Moro ${ }^{1}$, Anita Holzinger ${ }^{3}$, \\ Alessandra Mereu ${ }^{1}$, Antonio Preti ${ }^{1}$ and Mauro Giovanni Carta ${ }^{1}$
}

\author{
${ }^{I}$ Department of Public Health and Clinical and Molecular Medicine, University of Cagliari, Italy; ${ }^{2}$ Radboud University \\ Nijmegen, The Nederlands; ${ }^{3}$ Medical University of Vienna, Clinical Institute of Pathology, Austria
}

\begin{abstract}
Background: The school setting may be the optimal context for early screening of and intervention on child mental health problems, because of its large reach and intertwinement with various participants (child, teacher, parent, other community services). But this setting also exposes children to the risk of stigma, peer rejection and social exclusion. This systematic literature review investigates the efficacy of mental health interventions addressed to children and adolescents in school settings, and it evaluates which programs explicitly take into account social inclusion indicators. Method: Only randomized controlled trials conducted on clinical populations of students and carried out in school settings were selected: 27 studies overall. Most studies applied group Cognitive Behavioural Therapy or Interpersonal Psychotherapy. Results: Findings were suggestive of the effectiveness of school-based intervention programs in reducing symptoms of most mental disorders. Some evidence was found about the idea that effective studies on clinical populations may promote the social inclusion of children with an ongoing mental disorder and avoid the risk of being highly stigmatized. Conclusion: School programs are still needed that implement standardized models with verifiable and evidence-based practices involving the whole school community.
\end{abstract}

Keywords: Educational context, mental health, school.

\section{INTRODUCTION}

Schools are considered the ideal setting for the implementation of mental health treatment interventions, for several reasons.

Since the vast majority of children attends school and spends a considerable amount of time in school, the school is not only a setting for the early detection of children at risk of mental health disorders, but it also creates numerous possibilities to target these children with early interventions. Furthermore, the school provides a complex, far-reaching network of community, parents, teachers and peers who, when involved, have a large potential of influencing child development.

School-based screening or treatment programs for common mental disorders can raise complex issues as well. One often feared risk is the potential over-diagnosing of students with the risk of stigmatizing them with a life-long label, damaging their social interactions and peer acceptance. Indeed, stigma and discrimination behaviour towards mental health disorders have been observed in even the youngest school children $[1,2]$.

To avoid the risk of stigmatization, there is some agreement that school-based screening and intervention programs

\footnotetext{
*Address correspondence to this author at the Department of Public Health and Clinical and Molecular Medicine. University of Cagliari, Italy;

Tel: +39/070/6093495; E-mail: giuliaci@hotmail.com
}

should not merely address clinical or cognition-based problems, but also include experiential social activities, engage students' feelings and behaviour thus facilitating their interaction with others, and develop their social skills [3, 4]. Within the worldwide call to eliminate and prevent mental health stigma and its antecedents [5], programs aimed at facilitating integration of children with psychiatric problems in the community were developed and tested.

Social competence is an important aspect in youth development and can be defined as the ability to form and maintain positive relationships and pro-social styles of interaction, and the ability to read social situations and to interpret them correctly. The absence of pro-social strategies often leads to dislike by peers, hence social exclusion. For children with a mental disease, peer-rejection at school can prompt or exacerbate antisocial development, while acceptance by peers could buffer the effects of dysfunctional behaviors $[6,7]$. Interventions effectiveness could therefore benefit from the inclusion of strategies that strengthen social competence and stimulate peer acceptance in the school setting and in the community $[6,8,9]$, and vouch for a functional network and community.

To create efficient functional networks and communities, school-based mental health activity and intervention programs increasingly involve families and school personnel in treatment. There is some evidence that positive interaction of families and school staff helps to achieve an overall functional school climate. Providers and families who work col- 
laboratively for a student are more likely to win the student's collaboration, which can result in positive role modelling $[10,11]$. Whole-school interventions have been emphasised recently. Sugai and Horner (2002) [12] described this system-based approach as a model that incorporates researchvalidated procedures and outcomes, consistent with international policies and guidelines about the best school practices. It also includes positive reinforcement and skill building approaches, prevents stress, and integrates all the elements of the school culture engaging students, teachers, administrators, and parents in practices.

Research and practices demonstrate that whole-school discipline programs, involving the school and its surrounding community as a unit of change, can be effective in reducing dysfunctional behaviours, preventing mental health problems, and contributing to a better students' performance [1216].

Educational achievement can materialize as academic success, and also in successful social relationships and integration at school. Engaging teachers into proactive and cooperative classroom management can produce positive environments that encourage and reinforce functional classroom behavior [4, 11, 17-19]. To reduce the risk for children with a mental illness to have poor performance and a stressful social experience at school, resulting in potential exacerbation of the mental illness, practices need to improve their everyday psychosocial functioning in both school and home settings, involving the pupil's parents, teachers and community in school interventions [8].

\section{OBJECTIVES}

The main purpose of this study was to conduct a systematic review of school-based treatments or programs focusing on the integration of students with psychiatric diagnosis in the classroom. The concept of Integration comprises social inclusion dimensions, social skills, the sense of belonging to a group, inclusion in the school network, and quality relationships with peers: this dimension was labelled as "ingroup" [20]. Its opposite, conceived as a dichotomous construct, could be constituted by discrimination, the presence of stigmas, peer dysfunctional relationships, social exclusion, social anxiety, and low participation in school and recreational activities. The effectiveness of the interventions will be evaluated, while social outcome or social skills will be assessed as well as possible indicators of social inclusion/exclusion variables. Given the low number of studies specifically aimed at integration, authors decided to review all the studies involving clinical populations of students, ensuring the overall effectiveness of the interventions and assessing social outcomes and their changes after treatments apart.

\section{METHODS}

\section{Inclusion and Exclusion Criteria}

As a requirement of the search criteria, all the selected studies were based on randomized controlled trials (RCTs), which is considered the gold standard methodology to assess a program's effectiveness. More specifically, to be included studies had to involve a school-age (3-18 years old) clinical sample; they must have been carried out in school settings and verified through clinical, psycho-social, learning or academic skills outcomes. Only studies written in English and published from 2000 to 2014 were included. Primary and secondary prevention programs on at-risk populations chosen according to socio-demographic variables, and interventions focusing on addictions and substance abuse were excluded.

\section{Search Strategy and Study Selection}

We searched PubMed, and the Google Scholar databases using the following key words: "mental health, educational context, school"; no additional filters were used. This initial search yielded 17,700 hits, while seven additional studies were retrieved by searching on included articles' references or following indication by expert authors. Eventually, 1,090 abstracts written in English were examined to determine whether they met the specific inclusion and exclusion criteria. Overall sixty full-text articles were assessed for eligibility. Out of these, five studies were excluded because they were preliminary protocol descriptions or feasibility studies; one study was a psychometric validation of a questionnaire; nineteen studies did not refer to a randomized controlled trial; two studies referred to prevention programs applied to non-clinical samples; four studies referred to treatment other than school-based; two studies were reports of pharmacological treatments and did not refer to a psychosocial intervention (Fig. (1): the flowchart according to the Preferred Reporting Items for Systematic Reviews and Meta-Analyses, PRISMA).

\section{RESULT}

\section{General Description of Included Studies}

Overall, 27 RCT papers met the inclusion criteria and were included in the qualitative synthesis.

These school-based interventions differed among them in terms of the role played by teachers and parents in the treatment. In some studies, treatment was targeted at parents or teachers and the outcomes were measured on school children. In other studies, students, teachers and parents were equally involved in the treatment; other studies instead delivered treatment to pupils only. Presentation of results and interpretation of findings was divided in two parts: schoolbased programs that actively involved parents or teachers in the interventions, and interventions aimed at students only. The main outcomes that revealed a statistical significance were reported (see Table 1, 2). Table 3 describes more in detail the psychometric instruments used to test social variables of social inclusion and their variation in relation to the intervention.

\section{School-based Interventions on Clinical Samples with Ac- tive Participation of Teachers and/or Parents}

Three of the parent- and teacher-training program treatments concerned children samples with Attention Deficit Hyperactivity Disorder (ADHD) (Table 1). Two studies concerned Disruptive, Impulse-Control, and Conduct Disorders; more specifically, one dealt with oppositional defiant disorder (ODD) and the other with both Conduct Disorder (CD) and ODD children samples. One study followed a parenting 


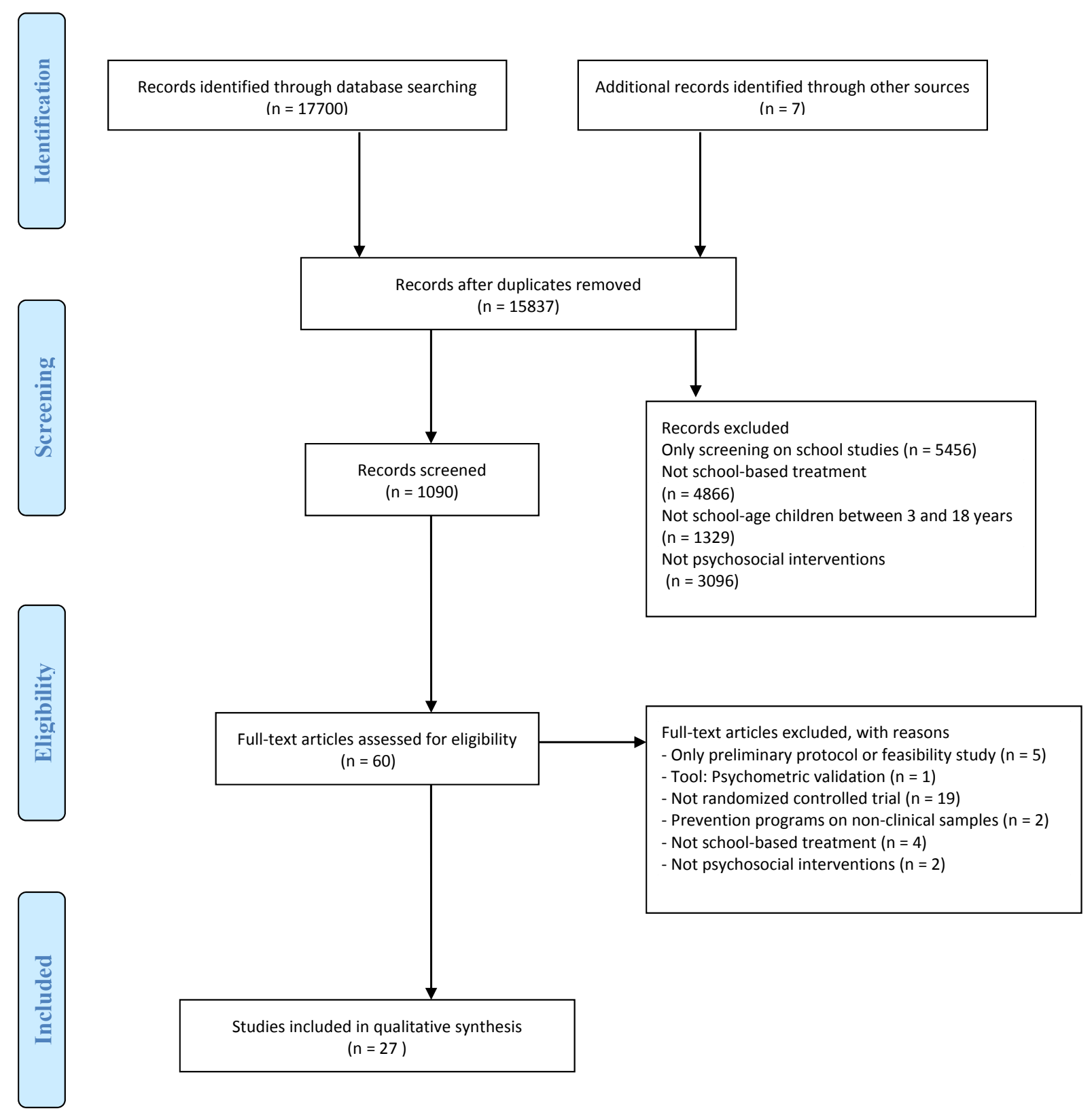

Fig. (1). Prisma flow diagram.

program for the management of children with Intellectual Disability, one was a Teachers' educational program on common mental disorders (including Depression, Anxiety, Psychosis, Behavioural disorders) and three studies concerned Psycho-educational and Cognitive Behavioural Therapy (CBT) group treatments on a sample of children with Anxiety disorders, with participation of both parents and teachers.

\section{School-based Interventions on Clinical Samples Target- ing Pupils Only}

Two studies concerned Disruptive, Impulse-Control, and Conduct Disorders, specifically one was on Conduct Disorder (CD), and the other had a children sample with behavioural problems as a specification of Conduct Disorders (Table 2).
One study investigated the efficacy of school-based interventions on a sample of ADHD pupils; two studies investigated the effectiveness of treatment on both Anxiety and Depression disorders in a clinical sample of school children, seven studies focused solely on mood disorder school programs, six studies focused on the treatment of Depression disorder, and one on Emotional distress; four studies were about Post-Traumatic Stress Disorder (PTSD) programs; and one study was a treatment focused on Anxiety Disorders in a clinical school children sample.

\section{Treatment Effectiveness of School-based Interventions on Clinical Samples with Active Participation of Teach- ers and/or Parents}

The overall findings on school-based treatment of clinical samples with the active participation of teachers and/or 
Table 1. Efficacy of school-based interventions on clinical samples targeting pupils with active participation of teachers and/or parents.

\begin{tabular}{|c|c|c|c|c|c|c|c|}
\hline Study & Country & Diagnosis & $\begin{array}{l}\text { Type of } \\
\text { program/FU }\end{array}$ & $\begin{array}{l}\text { Sample size } \\
\text { and group }\end{array}$ & Measures/outcome & Result & Social outcome \\
\hline $\begin{array}{l}\text { Ostberg } \\
\text { et al. } \\
2012\end{array}$ & Sweden & $\begin{array}{l}\text { Attention deficit } \\
\text { hyperactivity } \\
\text { disorder } \\
\text { (ADHD) }\end{array}$ & $\begin{array}{l}\text { Parent and teacher } \\
\text { manual-based group } \\
\text { training } \\
\text { Program } \\
\text { 3-month T3 }\end{array}$ & $\begin{array}{l}\text { Children Treatment } \\
\text { group TG }(n=46) \\
\text { Children control group } \\
\text { CG }(n=46) \\
61 \text { par. } / 68 \text { teachers } \\
\text { Age } m=10.95\end{array}$ & $\begin{array}{l}\text { ADHD Rating Scale } \\
\text { ODD symptoms were } \\
\text { measured by the eight } \\
\text { DSM-IV criteria } \\
\text { The Strengths and } \\
\text { Difficulties Question- } \\
\text { naire (SDQ) }\end{array}$ & $\begin{array}{l}\text { (Assessed by parents } \\
\text { and Teachers) } \\
\text { ADHD } \\
\text { Rating Scale } \\
\text { TG } \downarrow \text { p }<0.01 \text { at T3 } \\
\text { ODD } \\
\text { symptoms } \\
\text { TG } \downarrow \text { p }<0.05 \text { at T3 } \\
\text { SDQ } \\
\text { TG } \downarrow \text { p }<0.05 \text {. at T3 } \\
\text { Stronger efficacy } \\
\text { assessed by parents }\end{array}$ & $\begin{array}{l}\text { SDQ : } \\
\text { Peer relationship prob- } \\
\text { lems } \\
(5 \text { items }) \\
\text { Prosocial behaviour } \\
(5 \text { items })\end{array}$ \\
\hline $\begin{array}{l}\text { Sayal et al. } \\
2010\end{array}$ & $\begin{array}{l}\text { United } \\
\text { King- } \\
\text { domUni }\end{array}$ & $\begin{array}{l}\text { Attention deficit } \\
\text { hyperactivity } \\
\text { disorder } \\
\text { (ADHD) }\end{array}$ & $\begin{array}{l}\text { Early school-based } \\
\text { screening and educa- } \\
\text { tional intervention } \\
5 \text {-year follow-up }\end{array}$ & $\begin{array}{l}\text { Children group book } \\
\text { only } \\
\text { TG }(n=81) \\
\text { Children group identifi- } \\
\text { cation only } \\
\text { CI ( } n=114) \\
\text { Children group book and } \\
\text { identification } \\
\text { TGI ( }=99) \\
\text { Children group no } \\
\text { intervention } \\
\text { CG }(n=84) \\
\text { Age } m=7.5\end{array}$ & $\begin{array}{l}\text { The Strengths and } \\
\text { Difficulties Question- } \\
\text { naire (SDQ) }\end{array}$ & $\begin{array}{l}\text { (Assessed by parents } \\
\text { and Teachers) } \\
\text { SDQ } \leftrightarrow \\
\text { Stronger efficacy } \\
\text { assessed by Teachers }\end{array}$ & $\begin{array}{l}\text { SDQ : } \\
\text { Peer relationship prob- } \\
\text { lems } \\
(5 \text { items }) \\
\text { Prosocial behaviour } \\
(5 \text { items })\end{array}$ \\
\hline $\begin{array}{l}\text { Murray } \\
\text { et al. } \\
2011\end{array}$ & $\begin{array}{l}\text { United } \\
\text { States }\end{array}$ & $\begin{array}{l}\text { Attention deficit } \\
\text { hyperactivity } \\
\text { disorder (ADHD) }\end{array}$ & $\begin{array}{l}\text { Teacher Management } \\
\text { Practices for children } \\
\text { with ADHD } \\
\text { No follow-up }\end{array}$ & $\begin{array}{l}\text { Teachers }(\mathrm{n}=36) \\
\text { Children intervention } \\
\text { group } \\
\text { TG }(\mathrm{n}=46) \\
\text { Attention training } \\
\text { control group CG }(\mathrm{n}=46) \\
\text { Age } \mathrm{m}=6.5\end{array}$ & $\begin{array}{l}\text { Teacher Management } \\
\text { Questionnaire } \\
\text { (TMQ) }\end{array}$ & $\begin{array}{l}\text { (Assessed by Teacher) } \\
\text { TMQ subscales: } \\
\text { Environmental modifica- } \\
\text { tion } \\
\text { TG } \uparrow p<0.001 \\
\text { Behavior modification } \\
\text { TG } \uparrow p<0.05 \\
\text { Assignment modi- } \\
\text { fication } \\
\text { TG } \uparrow p<0.001 \\
\text { Structure and organiza- } \\
\text { tion } \leftrightarrow \\
\text { Instructional modifica- } \\
\text { tions } \leftrightarrow\end{array}$ & \\
\hline $\begin{array}{l}\text { Drugli } \\
\text { et al. } \\
2006\end{array}$ & Norway & $\begin{array}{l}\text { Oppositional } \\
\text { defiant disorder } \\
\text { (ODD) }\end{array}$ & $\begin{array}{l}\text { Parent training combined } \\
\text { with child therapy on } \\
\text { positive discipline } \\
\text { strategies, coping and } \\
\text { social skills, conflict } \\
\text { resolution, playing and } \\
\text { cooperation with peers. } \\
\text { 1-year follow-up }\end{array}$ & $\begin{array}{l}\text { Children parent training } \\
\text { group PT }(\mathrm{n}=47) \\
\text { Children parent training } \\
\text { and therapy group } \\
\mathrm{PT}+\mathrm{CT} \\
(\mathrm{n}=52) \\
\text { Waiting-list group WLC } \\
(\mathrm{n}=28 \text { families }) \\
\text { Age } \mathrm{m}=6\end{array}$ & $\begin{array}{l}\text { Teacher Report Form } \\
\text { (TRF) } \\
\text { Preschool Behavior } \\
\text { Questionnaire } \\
\text { (PBQ) } \\
\text { Social Competence and } \\
\text { Behavior Evaluation } \\
\text { (SCBE) } \\
\text { The Wally Child Social } \\
\text { Problem-Solving Detec- } \\
\text { tive Game (WALLY) } \\
\text { Student-Teacher } \\
\text { Relationship Scale } \\
\text { (STRS) }\end{array}$ & $\begin{array}{l}\text { Assessed by parents } \\
\text { and Teachers) } \\
\text { TRF } \\
\text { Aggression problems at } \\
\text { post-treatment } \\
\text { PT+CT vs PT } \downarrow \text { p }<0.05 \\
\text { PT+CT vs t WLC } \downarrow \\
\text { p }<0.01 \\
\text { Aggression problems } \\
\text { across post-treatment } \\
\text { and follow-up } \\
\text { PT+CT } \uparrow \text { p }<0.01 \\
\text { PBQ } \\
\text { clinical level at post- } \\
\text { treatment } \\
\text { PT }+ \text { CT } \downarrow \text { p }<0.05 \\
\text { clinical level at follow- } \\
\text { up } \\
\text { PT }+ \text { CT } \uparrow \mathrm{p}<0.05 \\
\text { WALLY } \\
\text { social strategies at post- } \\
\text { treatment PT }+ \text { CT } \uparrow \\
\text { p }<0.001 \\
\text { social strategies at } \\
\text { follow-up PT } \uparrow \mathrm{p}<0.05 \\
\text { SCBE } \leftrightarrow \\
\text { STRS } \leftrightarrow\end{array}$ & $\begin{array}{l}\text { WALLY } \\
\text { SCBE } \\
\text { STRS }\end{array}$ \\
\hline
\end{tabular}


(Table 1) contd....

\begin{tabular}{|c|c|c|c|c|c|c|c|}
\hline Study & Country & Diagnosis & $\begin{array}{l}\text { Type of } \\
\text { program/FU }\end{array}$ & $\begin{array}{l}\text { Sample size } \\
\text { and group }\end{array}$ & Measures/outcome & Result & Social outcome \\
\hline $\begin{array}{l}\text { Drugli } \\
\text { et al. } \\
2007\end{array}$ & Norway & $\begin{array}{l}\text { Conduct disorders } \\
\text { (CD) } \\
\text { Oppositional } \\
\text { defiant disorder } \\
\text { (ODD) }\end{array}$ & $\begin{array}{l}\text { Parent training combined } \\
\text { with child therapy on } \\
\text { positive discipline } \\
\text { strategies, coping and } \\
\text { social skills, conflict } \\
\text { resolution, playing and } \\
\text { cooperation with peers. } \\
\text { 1-year follow-up }\end{array}$ & $\begin{array}{l}\text { Children in parent } \\
\text { training and therapy } \\
\text { group PT }+\mathrm{CT} \\
(\mathrm{n}=52) \\
\text { Children in parent } \\
\text { training group PT } \\
\text { (control) (n=47) } \\
\text { Waiting-list group WLC } \\
(\mathrm{n}=28) \\
\text { Age } \mathrm{m}=6\end{array}$ & $\begin{array}{l}\text { The Child Behaviour } \\
\text { Checklist (CBCL) } \\
\text { The Wally Child Social } \\
\text { Problem-Solving Detec- } \\
\text { tive Game (WALLY) } \\
\text { The Child Loneliness } \\
\text { and Social Dissatisfac- } \\
\text { tion Questionnaire } \\
\text { (LSC) } \\
\text { Social Competence and } \\
\text { Behaviour Evaluation } \\
\text { (SCBE) }\end{array}$ & $\begin{array}{l}\text { (Assessed by parents } \\
\text { and Teachers) } \\
\text { CBCL (father reports) } \\
\text { from post-treatment and } \\
\text { maintained across } \\
\text { follow-up } \\
\text { PT }+ \text { CT } \uparrow \mathrm{p}<0.01 \\
\text { PT } \uparrow \mathrm{p}<0.05 \\
\text { CBCL (mother ratings) } \\
\text { from post-treatment and } \\
\text { maintained across } \\
\text { follow-up } \\
\text { PT }+ \text { CT } \uparrow p<0.05 \\
\text { WALLY } \\
\text { Number of pro-social } \\
\text { strategies used and } \\
\text { maintained across } \\
\text { follow-up } \\
\text { PT }+ \text { CT } \uparrow p<0.01 \\
\text { SCBE } \leftrightarrow \\
\text { LSC } \leftrightarrow\end{array}$ & $\begin{array}{l}\text { CBCL } \\
\text { Social competence } \\
\text { WALLY } \\
\text { LSC } \\
\text { SCBE }\end{array}$ \\
\hline $\begin{array}{l}\text { Hand, et al. } \\
2013\end{array}$ & Ireland & $\begin{array}{l}\text { ID } \\
\text { Intellectual } \\
\text { Disability }\end{array}$ & $\begin{array}{l}\text { Evidence-based parent- } \\
\text { ing programs based on } \\
\text { social learning models } \\
\text { No follow-up }\end{array}$ & $\begin{array}{l}\text { Treatment group partici- } \\
\text { pants } \\
\text { TG } \\
(\mathrm{n}=16) \\
\text { Control group partici- } \\
\text { pants CG } \\
(\mathrm{n}=13) \\
42 \text { parents } \\
\text { Age } \mathrm{m}=9\end{array}$ & $\begin{array}{l}\text { The Strengths and } \\
\text { Difficulties Question- } \\
\text { naire (SDQ) } \\
\text { The Parenting } \\
\text { Stress Index (PSI) } \\
\text { The Kansas Parent } \\
\text { Satisfaction Scale (KPS) } \\
\text { Parent identified per- } \\
\text { sonal and child-related } \\
\text { goals. }\end{array}$ & $\begin{array}{l}\text { (Assessed by parents) } \\
\text { SDQ: (Time } \times \text { Group) } \\
\text { Total difficulties sub- } \\
\text { scales } \\
\text { TG } \downarrow \text { p }<0.007 \\
\text { Conduct problems } \\
\text { subscales } \\
\text { TG } \downarrow \text { p }<0.027 \\
\text { Hyperactivity } \leftrightarrow \\
\text { Emotional problems } \leftrightarrow \\
\text { Peer problems } \leftrightarrow \\
\text { Pro-social behaviour } \leftrightarrow \\
\text { Time factor } \\
\text { Total difficulties } \\
\text { TG } \downarrow p=0.003 \\
\text { Conduct problems } \\
\text { TG } \downarrow p=0.009 \\
\text { PSI stress Index (Time } \\
\times \text { Group) } \\
\text { total score } \\
\text { TG } \downarrow p<0.01 \\
\text { Total } \\
\text { TG } \downarrow p<0.01 \text { ). } \\
\text { (Time factor) } \\
\text { PSI Total score } \\
\text { TG } \downarrow p<0.001 \\
\text { Parent Distress } \\
\text { TG } \downarrow p=0.002 \\
\text { Parent-child relationship } \\
\text { difficulties } \\
\text { TG } \downarrow p=0.004 \\
\text { Difficult child measure } \\
\leftrightarrow \\
\text { KPS Satisfaction Scale } \\
\leftrightarrow \\
\text { Parent defined child- } \\
\text { related goals } \\
\text { (Time } \times \text { Group) } \\
\text { child-related goals } \\
\text { TG } \uparrow \mathrm{p}<0.001 \text {. } \\
\text { (Time effect) } \\
\text { TG v sCG } \uparrow \mathrm{p}<0.001\end{array}$ & $\begin{array}{l}\text { SDQ : } \\
\text { Peer relationship prob- } \\
\text { lems } \\
(5 \text { items }) \\
\text { Prosocial behaviour } \\
(5 \text { items })\end{array}$ \\
\hline
\end{tabular}


(Table 1) contd....

\begin{tabular}{|c|c|c|c|c|c|c|c|}
\hline Study & Country & Diagnosis & $\begin{array}{l}\text { Type of } \\
\text { program/FU }\end{array}$ & $\begin{array}{l}\text { Sample size } \\
\text { and group }\end{array}$ & Measures/outcome & Result & Social outcome \\
\hline $\begin{array}{l}\text { Jorm, et al. } \\
2010\end{array}$ & Australia & $\begin{array}{l}\text { Mental disorders } \\
\text { (Depression, Anxi } \\
\text { ety, } \\
\text { Psychosis, } \\
\text { Behavioural } \\
\text { disorders) }\end{array}$ & $\begin{array}{l}\text { Teachers educational } \\
\text { programs on common } \\
\text { mental disorders in } \\
\text { Adolescents and student } \\
\text { welfare } \\
6 \text { months follow-up }\end{array}$ & $\begin{array}{l}\text { Teachers Intervention } \\
\text { group TIG ( } \mathrm{n}=221) \\
\text { Teachers Control group } \\
\text { TCG }(\mathrm{n}=106) \\
\text { Students in intervention } \\
\text { group SIG }(\mathrm{n}=982) \\
\text { Students Control group } \\
\text { SCG }(\mathrm{n}=651) \\
\text { Age } \mathrm{m}=9\end{array}$ & $\begin{array}{l}\text { Teacher outcomes: } \\
\text { mental health knowledge } \\
\text { Personal stigma items: } \\
\% \\
\text { strongly disagree } \\
\text { Perceived stigma items: } \\
\% \geq \text { agree } \\
\text { Help given towards } \\
\text { students: \% } \geq \text { occasion- } \\
\text { ally } \\
\text { Confidence in helping } \\
\text { students and staff with } \\
\text { mental health problems } \\
\% \geq \text { quite a bit } \\
\text { School policies on } \\
\text { student mental health } \\
\text { Interacting with col- } \\
\text { leagues: \% } \geq \\
\text { occasionally } \\
\text { Seeking Additional } \\
\text { Mental health informa- } \\
\text { tion: \% } \geq \text { occasionally } \\
\text { Teacher mental health } \\
\text { Student outcome: } \\
\text { Mental health knowl- } \\
\text { edge } \\
\text { Beliefs and intentions } \\
\text { about where to seek help } \\
\text { for depression } \\
\text { Personal stigma: \% } \\
\text { strongly disagree } \\
\text { Perceived stigma: \% } \geq \\
\text { agree } \\
\text { Help received from } \\
\text { teacher } \\
\text { Student Mental Health }\end{array}$ & \begin{tabular}{|l} 
(Assessed by Clini- \\
cians) \\
(Teacher) \\
knowledge \\
TIG $\uparrow p<0.001$ and \\
maintained at follow-up \\
P $<0.001$. \\
Perceived stigma (OR) \\
TIG $\uparrow p=0.031$ and \\
maintained at follow -up \\
See other people as \\
reluctant to disclose \\
TIG $\uparrow p=0.041$ and \\
maintained at follow -up \\
Intentions towards \\
helping students $($ OR) \\
Teachers more likely to \\
discuss their concerns \\
with another teacher \\
TIG $\uparrow p=0.013$ and \\
maintained at follow -up \\
Discuss their concerns \\
with a counsellor (OR) \\
TIG $\uparrow p=0.023$ and \\
maintained at follow -up \\
Have a conversation \\
with the student $($ OR) \\
TIG $\uparrow p=0.162$ and \\
maintained at follow -up \\
School policy of mental \\
health (OR) \\
TIG $\uparrow p=0.019$ and \\
maintained at follow -up \\
Policy had been im- \\
plemented in the \\
previous month (OR) \\
TIG $\uparrow p=0.070$ and \\
maintained at follow-up \\
(Student) \\
Mental health knowl- \\
edge \\
Report that they received \\
information about \\
mental health problems \\
SIG $\uparrow P<0.001$ \\
Beliefs and intentions \\
about where to seek \\
help for depression \\
(Student outcome) $\leftrightarrow$ \\
Personal stigma: \% \\
strongly disagree \\
(Student outcome) $\leftrightarrow$ \\
Perceived stigma: \% $\geq$ \\
agree \\
Stigma perceived in \\
others (OR) \\
SIG $\uparrow p=0.006$. \\
Help received from \\
teacher $\leftrightarrow$ \\
Student Mental Health \\
$\leftrightarrow$ \\
$\leftrightarrow$
\end{tabular} & $\begin{array}{l}\text { Students } \\
\text { Personal Stigma Items: } \\
\% \\
\text { Strongly Disagree } \\
\text { Perceived Stigma Items: } \\
\% \geq \\
\text { Agree } \\
\text { Teachers } \\
\text { Personal Stigma: \% } \\
\text { Strongly Disagree } \\
\text { Perceived Stigma: \% } \geq \\
\text { Agree }\end{array}$ \\
\hline
\end{tabular}


(Table 1) contd....

\begin{tabular}{|c|c|c|c|c|c|c|c|}
\hline Study & Country & Diagnosis & $\begin{array}{l}\text { Type of } \\
\text { program/FU }\end{array}$ & $\begin{array}{l}\text { Sample size } \\
\text { and group }\end{array}$ & Measures/outcome & Result & Social outcome \\
\hline $\begin{array}{l}\text { Mifsud, } \\
\text { et al. } \\
2005\end{array}$ & Australia & Anxiety & $\begin{array}{l}\text { Cognitive Behavioral } \\
\text { Therapy Treatment } \\
\text { groups ( } 8-10 \text { children) } \\
\text { with parents collabora- } \\
\text { tion } \\
\text { 4-Month Follow-up }\end{array}$ & $\begin{array}{l}\text { Treatment group TG }(\mathrm{n}= \\
\text { 92) } \\
\text { Active intervention } \\
\text { waitlist control } \\
\text { CG } \\
\text { (num not reported) } \\
\text { Age } m=9.5\end{array}$ & $\begin{array}{l}\text { Spence Children's } \\
\text { Anxiety Scale (SCAS) } \\
\text { Children's Automatic } \\
\text { Thoughts Scale (CATS) } \\
\text { Spence Children's } \\
\text { Anxiety Scale-Parent } \\
\text { Version (SCAS-P) } \\
\text { (Sec.) Child Behavior } \\
\text { Checklist-(CBCL) } \\
\text { Teacher Rep. }\end{array}$ & $\begin{array}{l}\text { (Assessed by Clini- } \\
\text { cians) } \\
\text { SCAS } \\
\text { TG } \downarrow p<0.005 \text { and } \\
\text { maintained at follow-up } \\
\text { CATS } \\
\text { TG } \downarrow p=.001 \text { and } \\
\text { maintained at follow-up } \\
\text { SCAS-P } \leftrightarrow \\
\text { CBCL } \leftrightarrow\end{array}$ & $\begin{array}{l}\text { SCAS: } \\
\text { sub-scale s } \\
\text { Social phobia } \\
\text { CATS: } \\
\text { (9 items) } \\
\text { CBCL: } \\
\text { Social problems }\end{array}$ \\
\hline $\begin{array}{l}\text { Masia et al. } \\
2005\end{array}$ & $\begin{array}{l}\text { United } \\
\text { States }\end{array}$ & Anxiety & $\begin{array}{l}\text { Skills training interven- } \\
\text { tion on adolescents and } \\
\text { participation of parents } \\
\text { and teachers on psycho- } \\
\text { education groups } \\
\text { 9-month } \\
\text { follow-up }\end{array}$ & $\begin{array}{l}\text { Intervention group } \\
(\mathrm{SASS}) \mathrm{TG} \\
(\mathrm{n}=21) \\
\text { Control group } \mathrm{WL} \\
(\mathrm{n}=17) \\
\text { Age } \mathrm{m}=14.8\end{array}$ & $\begin{array}{l}\text { Self-Report Inventories } \\
\text { (ADIS-PC) Severity } \\
\text { Social Phobic Disorders } \\
\text { Severity and Change } \\
\text { Form (SPDSCF) } \\
\text { Liebowitz Social Anxi- } \\
\text { ety Scale for Children } \\
\text { and Adolescents (LSAS- } \\
\text { CA) } \\
\text { Children's Global } \\
\text { Assessment } \\
\text { Scale(CGAS) } \\
\text { Social Phobia and } \\
\text { Anxiety Inventory for } \\
\text { Children } \\
\text { (SPAI-C) } \\
\text { Social Anxiety Scale for } \\
\text { Adolescents (SAS-A) } \\
\text { Self-Report Inventories } \\
\text { Loneliness Scale } \\
\text { Parent Report: } \\
\text { Social Anxiety Scale for } \\
\text { Adolescents (SAS-AP) }\end{array}$ & 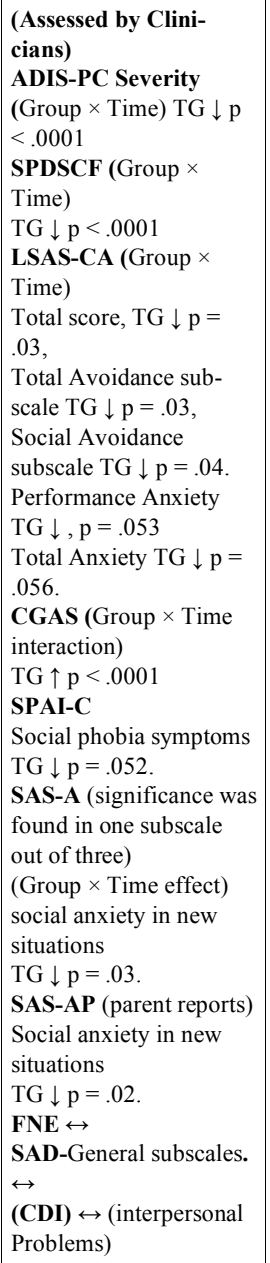 & \begin{tabular}{|l} 
SPDSCF \\
LSAS-CA \\
SPAI-C \\
SAS-A (AP) \\
CDI: \\
(interpersonal Problems) \\
Self-Report Inventories \\
Loneliness Scale \\
ADIS-PC: \\
social phobia items \\
C-GAS: Children's \\
global assessment scale \\
(the area of interaction \\
with with friends)
\end{tabular} \\
\hline $\begin{array}{l}\text { Bernstein, } \\
\text { et al. } \\
2005\end{array}$ & $\begin{array}{l}\text { United } \\
\text { States }\end{array}$ & Anxiety & $\begin{array}{l}\text { Group cognitive- } \\
\text { behavioral therapy } \\
\text { (CBT) for children plus } \\
\text { parent training } \\
\text { Group } \\
\text { 6-month follow-up. }\end{array}$ & $\begin{array}{l}\text { Treatment group CBT } \\
\text { for children TG }(\mathrm{n}=17), \\
\text { Treatment group CBT } \\
\text { for children plus parent } \\
\text { training TPP } \\
(\mathrm{n}=20) \\
\text { No-treatment control } \\
\text { group } \\
(\mathrm{n}=24) \\
\text { Age } \mathrm{m}=9.0\end{array}$ & \begin{tabular}{|l|} 
Child and Parent Inter- \\
view Schedules (ADIS) \\
Multidimensional \\
Anxiety Scale for \\
Children (MASC-C) \\
Screen for Child Anxiety \\
Related Emotional \\
Disorders (SCARED) \\
Clinical Global Impres- \\
sions (CGI) \\
Services Questionnaire \\
(developed for use in \\
this study)
\end{tabular} & $\begin{array}{l}\text { (Assessed by Parent } \\
\text { and Clinicians) } \\
\text { ADIS } \\
\text { Composite CSR } \\
\text { TG+TPP } \uparrow \mathrm{p}=.03 \\
\text { TPP } \uparrow \mathrm{p}=.06 \\
\text { Child-Interview } \\
\text { TG+TPP } \uparrow \mathrm{p}=.045 \\
\text { CGI } \\
\text { TG+TPP } \uparrow \mathrm{p}=.06 \\
\text { TPP } \uparrow \mathrm{p}=.02 \text { ). } \\
\text { MASC }(\text { group } \times \text { time) } \\
\text { TG+TPP } \uparrow \mathrm{p}=.006 \\
\text { SCARED } \text { (group } \times \\
\text { time) } \\
\text { TG+TPP } \downarrow \mathrm{p}=.001 \\
\text { MASC Total } \\
\text { TPP } \uparrow \mathrm{p}=.02 . \\
\text { SCARED indic. } \\
\text { TPP } \uparrow \mathrm{p}=.000 .\end{array}$ & $\begin{array}{l}\text { ADIS: } \\
\text { social phobia items } \\
\text { MASC: } \\
\text { The Social Anxiety scale } \\
\text { Humiliation/Rejection } \\
\text { subscale }\end{array}$ \\
\hline
\end{tabular}


Table 2. Efficacy of school-based interventions on clinical samples targeting pupils only.

\begin{tabular}{|c|c|c|c|c|c|c|c|}
\hline Study & Country & Diagnosis & $\begin{array}{l}\text { Type of } \\
\text { program/FU }\end{array}$ & $\begin{array}{l}\text { Sample size } \\
\text { And group }\end{array}$ & Measures/outcome & Result & $\begin{array}{l}\text { Social } \\
\text { outcome }\end{array}$ \\
\hline $\begin{array}{l}\text { Hong, } \\
\text { et al. } \\
2011\end{array}$ & China & $\begin{array}{l}\text { Behavioral } \\
\text { problems }\end{array}$ & $\begin{array}{l}\text { Child Cogni- } \\
\text { tive-behavioral } \\
\text { intervention } \\
6 \text {-month follow } \\
\text { Up }\end{array}$ & $\begin{array}{l}\text { Treatment } \\
\text { group TG } \\
(\mathrm{n}=208) \\
\text { Control } \\
\text { group CG } \\
(\mathrm{n}=209) \\
\text { Age } \mathrm{m}=8\end{array}$ & $\begin{array}{l}\text { Child Behavior } \\
\text { Checklist. } \\
\text { (CBCL) } \\
\text { Total Behavior } \\
\text { Problem Scores }\end{array}$ & $\begin{array}{l}\text { (Assessed by Parents) } \\
\text { CBCL } \\
\text { Total behavior problem scores } \\
\text { TG } \downarrow p=.024 \\
\text { TG } \downarrow p=.001 \text { at } 6 \text {-month follow up } \\
\text { The levels of reported total behavior problems declined in } \\
\text { response to the intervention and remained lower than those in the } \\
\text { control group } 6 \text { months later }\end{array}$ & $\begin{array}{l}\text { CBCL: } \\
\text { Social prob- } \\
\text { lem scale }\end{array}$ \\
\hline $\begin{array}{l}\text { Leff, } \\
\text { et al. } \\
2009\end{array}$ & $\begin{array}{l}\text { United } \\
\text { States }\end{array}$ & $\begin{array}{l}\mathrm{CD} \\
\text { (Conduct } \\
\text { problems) }\end{array}$ & $\begin{array}{l}\text { Culturally- } \\
\text { adapted social } \\
\text { problem } \\
\text { solving/social } \\
\text { skills } \\
\text { intervention } \\
\text { No follow-up }\end{array}$ & $\begin{array}{l}\text { Intervention } \\
\text { group TG } \\
(n=21) \\
\text { Control } \\
\text { Group CG } \\
(n=11) \\
\text { Age } \mathrm{m}=12\end{array}$ & $\begin{array}{l}\text { The Children's Social } \\
\text { Behavior } \\
\text { Questionnaire (CSB) } \\
\text { Measure of Hostile } \\
\text { Attributional Bias } \\
\text { (HAB) with cartoon- } \\
\text { based version } \\
\text { Asher and Wheeler } \\
\text { Loneliness Scale } \\
\text { Children's Depres- } \\
\text { sion Inventory } \\
\text { (ALS) }\end{array}$ & $\begin{array}{l}\text { (Assessed by Peer, Teachers and Clinicians) } \\
\text { CSB } \\
\text { Teacher reports of relational aggression } \\
\text { TG } \downarrow \text { (moderate to large effect size of .74, Cohen, 1988) } \\
\text { Teacher ratings of peer likeability } \\
\text { TG } \uparrow(\text { very large effect size of } 1.73 \text {, Cohen, } 1988) \\
\text { HAB } \\
\text { TG } \downarrow \text { (very large effect size of .61, Cohen, 1988) } \\
\text { ALS } \\
\text { TG } \downarrow \text { (moderate effect size of .45. Cohen, } 1988\end{array}$ & $\begin{array}{l}\text { Peer nomina- } \\
\text { tion survey: } \\
\text { relational } \\
\text { aggression, } \\
\text { physical } \\
\text { aggression, } \\
\text { peer liking } \\
\text { hostile } \\
\text { Attributions } \\
\text { ALS } \\
\text { CSB }\end{array}$ \\
\hline $\begin{array}{l}\text { Owens } \\
\text { et al. } \\
2005\end{array}$ & $\begin{array}{l}\text { United } \\
\text { States }\end{array}$ & $\begin{array}{l}\text { ADHD } \\
\text { Attention } \\
\text { deficit } \\
\text { hyperactiv- } \\
\text { ity disorder } \\
\text { ODD } \\
\text { Oppositional } \\
\text { defiant } \\
\text { disorder } \\
\text { CD } \\
\text { Conduct } \\
\text { problems } \\
\text { DBD } \\
\text { Disruptive } \\
\text { Behavior } \\
\text { Disorders }\end{array}$ & $\begin{array}{l}\text { Behavioral } \\
\text { treatment } \\
\text { intervention } \\
9 \text { months } \\
\text { follow-up }\end{array}$ & $\begin{array}{l}\text { Treatment } \\
\text { group TG } \\
(\mathrm{n}=30) \\
\text { Waitlist Con- } \\
\text { trol group CG } \\
(\mathrm{n}=12) \\
\text { Age } \mathrm{m}=8.5\end{array}$ & $\begin{array}{l}\text { Disruptive Behavior } \\
\text { Disorders Struc- } \\
\text { tured Interview } \\
\text { (DBD) } \\
\text { Child Behavior } \\
\text { Checklist } \\
\text { (CBCL) } \\
\text { Impairment Rating } \\
\text { Scale } \\
\text { (IRS) }\end{array}$ & $\begin{array}{l}\text { (Assessed by Parents and Teachers) } \\
\text { DBD Rating Scale, } \\
\text { severity of hyperactivity and impulsivity TG } \downarrow \text { p }<.05 \\
\text { oppositional defiant behaviour TG } \downarrow \text { p }<.05 \\
\text { impairment in their peer relationships TG } \downarrow \text { p }<.05 \\
\text { CBCL, } \\
\text { aggressive symptomatology TG } \downarrow \text { p }<.10 \text {, } \\
\text { externalizing behavior problems TG } \downarrow \text { p }<.05 \\
\text { CD symptoms TG } \downarrow \text { p }<.10 \\
\text { total behavior problems TG } \downarrow \text { p }<.10\end{array}$ & $\begin{array}{l}\text { DBD } \\
\text { (peer relation- } \\
\text { ships) } \\
\text { IRS: } \\
\text { Peers relation } \\
\text { Sibling } \\
\text { relation } \\
\text { Parental } \\
\text { relation } \\
\text { CBCL: } \\
\text { ratings } \\
\text { Social }\end{array}$ \\
\hline $\begin{array}{l}\text { Cooper, } \\
\text { et al. } \\
2010\end{array}$ & $\begin{array}{l}\text { United } \\
\text { Kingdom }\end{array}$ & $\begin{array}{l}\text { Emotional } \\
\text { distress }\end{array}$ & $\begin{array}{l}\text { School-based } \\
\text { humanistic } \\
\text { counselling } \\
\text { intervention } \\
\text { no follow-up }\end{array}$ & $\begin{array}{l}\text { Counselling } \\
\text { group } \\
\text { TG } \\
(\mathrm{n}=13) \\
\text { Waiting list } \\
\text { group } \\
\text { WL } \\
(\mathrm{n}=14) \\
\text { Age } \mathrm{m}=14\end{array}$ & $\begin{array}{l}\text { The Self-Report } \\
\text { Strengths and Diffi- } \\
\text { culties Questionnaire } \\
\text { (SDQ) } \\
\text { (The emotional } \\
\text { symptoms subscale } \\
\text { of the SDQ) }\end{array}$ & $\begin{array}{l}\text { (Assessed by Clinicians) } \\
\text { SDQ-ES } \leftrightarrow\end{array}$ & $\begin{array}{l}\text { SDQ-PS: } \\
\text { prosocial } \\
\text { subscale } \\
\text { Secondary } \\
\text { outcome: } \\
\text { The Social } \\
\text { Inclusion } \\
\text { Questionnai- } \\
\text { re' (SIQ) }\end{array}$ \\
\hline $\begin{array}{l}\text { Mufson, } \\
\text { et al. } \\
2004\end{array}$ & $\begin{array}{l}\text { Unites } \\
\text { States }\end{array}$ & $\begin{array}{l}\text { Depres- } \\
\text { sion/Anxiety }\end{array}$ & $\begin{array}{l}\text { Interpersonal } \\
\text { psychotherapy } \\
\text { intervention } \\
16 \text { week } \\
\text { follow-up }\end{array}$ & $\begin{array}{l}\text { Treatment } \\
\text { group IPT-A, } \\
\text { TG } \\
\text { (n=34) } \\
\text { Treatment as } \\
\text { usual TAU, } \\
\text { CG } \\
(\mathrm{n}=29) \\
\text { Age } \mathrm{m}=15.1\end{array}$ & $\begin{array}{l}\text { Hamilton Rating } \\
\text { Scale for Depression } \\
\text { (HAMD) } \\
\text { Children's global } \\
\text { assessment scale } \\
\text { (C-GAS ) } \\
\text { Clinical Global } \\
\text { Impressions (CGI) } \\
\text { Social adjustment } \\
\text { scale-self report } \\
\text { (SAS-SR) }\end{array}$ & $\begin{array}{l}\text { (Assessed by Clinicians) } \\
\text { HAMD } \\
\text { TG } \downarrow p=.04 \text { and maintained at follow-up } \\
\text { C-GAS } \\
\text { TG } \uparrow p=.04 \text { (C-GAS trend to improvement at } 16 \text { weeks, } \mathrm{p}=0.06) \\
\text { CGI } \\
\text { Global functioning } \\
\text { TG } \uparrow p=0.03 \\
\text { mean CGI scores (improvement) } \\
\text { TG } \uparrow p=0.03 \\
\text { At } 16 \text { weeks slight effect size in global functioning } 0.51(95 \% \text { CI } \\
0.003 \text { to } 1.02) \\
\text { SAS-SR } \\
\text { social functioning mean } \mathrm{TG} \uparrow \mathrm{p}=0.01\end{array}$ & $\begin{array}{l}\text { C-GAS: } \\
\text { (interaction } \\
\text { with friends) } \\
\text { SAS-SR: } \\
\text { social adjust- } \\
\text { ment scale- } \\
\text { self report }\end{array}$ \\
\hline
\end{tabular}


(Table 2) contd....

\begin{tabular}{|c|c|c|c|c|c|c|c|}
\hline Study & Country & Diagnosis & $\begin{array}{l}\text { Type of } \\
\text { program/FU }\end{array}$ & $\begin{array}{l}\text { Sample size } \\
\text { And group }\end{array}$ & Measures/outcome & Result & $\begin{array}{l}\text { Social } \\
\text { outcome }\end{array}$ \\
\hline $\begin{array}{l}\text { O'Leary- } \\
\text { Barrett, } \\
\text { et al. } \\
2013\end{array}$ & $\begin{array}{l}\text { United } \\
\text { Kingdom }\end{array}$ & $\begin{array}{l}\text { Depression, } \\
\text { Anxiety, } \\
\text { Conduct } \\
\text { disorders }\end{array}$ & $\begin{array}{l}\text { Cognitive } \\
\text { behavioral } \\
\text { therapy inter- } \\
\text { vention } \\
2 \text { years follow- } \\
\text { up }\end{array}$ & $\begin{array}{l}\text { Treatment } \\
\text { group TG ( } \\
\mathrm{n}=694) \\
\text { Control group } \\
\text { CG }(\mathrm{n}=516) \\
\text { Age } \mathrm{m}=13.5\end{array}$ & $\begin{array}{l}\text { The Substance Use } \\
\text { Risk Profile Scale } \\
\text { (SURPS) } \\
\text { Brief symptoms } \\
\text { Inventory (BSI) } \\
\text { Strengths and Diffi- } \\
\text { culties Questionnaire } \\
\text { (SDQ) (conduct } \\
\text { subscale) }\end{array}$ & $\begin{array}{l}\text { (Assessed by Clinicians) } \\
\text { SURPS } \leftrightarrow \\
\text { BSI } \\
\text { depressive symptoms } \\
\text { TG } \downarrow \mathbf{p}<.05 \text { (over two years) } \\
\text { Suicidal ideation } \\
\text { TG } \downarrow \mathbf{p}<.02 \text { (over two years) } \\
\text { Anxiety symptoms } \\
\text { TG } \downarrow \mathbf{p}<.01 \text { (over two years) } \\
\text { Panic attacks } \leftrightarrow \\
\text { SDQ (conduct subscale) } \\
\text { TG } \downarrow \text { p }=.01 \text { (over two years) }\end{array}$ & \\
\hline $\begin{array}{l}\text { Stallard } \\
\text { et al. } \\
2012\end{array}$ & $\begin{array}{l}\text { United } \\
\text { kingdom }\end{array}$ & Depression & $\begin{array}{l}\text { Cognitive } \\
\text { behavioural } \\
\text { therapy } \\
12 \text { months } \\
\text { follow-up }\end{array}$ & $\begin{array}{l}\text { Usual school } \\
\text { inter-personal, } \\
\text { social, and } \\
\text { health } \\
\text { education } \\
\text { (PSHE) } \\
\text { UG (n=298) } \\
\text { Classroom } \\
\text { based CBT } \\
\text { group TG } \\
\text { ( n=392) } \\
\text { Attention } \\
\text { control group } \\
\text { CG (n=374) } \\
\text { Age m=14 }\end{array}$ & $\begin{array}{l}\text { Short mood and } \\
\text { feelings questionnaire } \\
\text { (SMFQ) }\end{array}$ & $\begin{array}{l}\text { (Assessed by Clinicians) } \\
\text { SMFQ } \leftrightarrow\end{array}$ & $\begin{array}{l}\text { Secondary } \\
\text { out.: } \\
\text { Rev. child } \\
\text { anx. and dep. } \\
\text { Scale } \\
\text { (RCADS) } \\
\text { Social fobia } \\
\text { scale }\end{array}$ \\
\hline $\begin{array}{l}\text { Stallard, } \\
\text { et al. } \\
2013\end{array}$ & $\begin{array}{l}\text { United } \\
\text { kingdom }\end{array}$ & Depression & $\begin{array}{l}\text { Classroom } \\
\text { based cognitive } \\
\text { behavioural } \\
\text { therapy } \\
12 \text { months } \\
\text { follow up }\end{array}$ & $\begin{array}{l}\text { Usual school } \\
\text { provision group } \\
\text { UG } \\
(\mathrm{n}=190) \\
\text { Attention } \\
\text { control per- } \\
\text { sonal, social, } \\
\text { and health } \\
\text { education } \\
\text { Interventions } \\
\text { PSHE group } \\
\text { CG } \\
(\mathrm{n}=179) \\
\text { Classroom- } \\
\text { based CBT } \\
\text { group TG } \\
(\mathrm{n}=344) \\
\text { Age m=14 }\end{array}$ & $\begin{array}{l}\text { Short Mood and } \\
\text { Feelings Question- } \\
\text { naire (SMFQ) } \\
\text { Cost-effectiveness: } \\
\text { incremental cost- } \\
\text { effectiveness ratios } \\
\text { (ICERs) } \\
\text { European Quality of } \\
\text { Life-5 Dimensions } \\
\text { score } \\
\text { (EQ-5D) } \\
\\
\end{array}$ & $\begin{array}{l}\text { (Assessed by Clinicians) } \\
\text { SMFQ } \leftrightarrow \\
\text { ICERs } \\
\text { Costs of interventions per child } \\
£ 41.96 \text { for classroom-based CBT; } £ 34.45 \text { for attention control } \\
\text { PSHE. } \\
\text { Fieller's method was used to obtain a parametric estimate of the } \\
95 \% \text { CI for the ICERs and construct the cost-effectiveness } \\
\text { acceptability curve, confirming that classroom-based CBT was } \\
\text { not cost-effective in the case of controls. } \\
\text { EQ-5D } \leftrightarrow\end{array}$ & $\begin{array}{l}\text { Secondary } \\
\text { outcomes: } \\
\text { Revised child } \\
\text { anx. and } \\
\text { dep.scale } \\
\text { (RCADS) } \\
\text { School } \\
\text { Connected- } \\
\text { ness subscale. } \\
\text { CATS } \\
\text { Social } \\
\text { phobia } \\
\text { subscale }\end{array}$ \\
\hline $\begin{array}{l}\text { Manas- } \\
\text { sis } \\
\text { et al. } \\
2010\end{array}$ & $\begin{array}{l}\text { United } \\
\text { States }\end{array}$ & Depression & $\begin{array}{l}\text { Classroom } \\
\text { based cognitive } \\
\text { behavioural } \\
\text { therapy } \\
12 \text { months } \\
\text { follow-up }\end{array}$ & $\begin{array}{l}\text { Cognitive } \\
\text { behavioural } \\
\text { therapy group } \\
\text { TG }(\mathrm{n}=78) \\
\text { Contrast } \\
\text { treatment at } \\
\text { usual CG } \\
(\mathrm{n}=70) \\
\text { Age } \mathrm{m}=9,5\end{array}$ & $\begin{array}{l}\text { Multidimensional } \\
\text { Anxiety Scale for } \\
\text { Children (MASC) } \\
\text { Children's Depres- } \\
\text { sion Inventory (CDI) }\end{array}$ & $\begin{array}{l}\text { (Assessed by Clinicians) } \\
\text { MASC } \\
\text { anxious symptoms } \\
\text { TG and in CG } \downarrow \mathrm{P}<.001 \\
\text { CDI } \\
\text { depressive symptoms } \\
\text { TG and in CG } \downarrow \mathrm{P}<.001\end{array}$ & $\begin{array}{l}\text { MASC: } \\
\text { The Social } \\
\text { Anxiety scale } \\
\text { Humiliation / } \\
\text { Rejection } \\
\text { subscale } \\
\text { CDI: } \\
\text { Interpersonal } \\
\text { Problems } \\
\text { Subscale }\end{array}$ \\
\hline $\begin{array}{l}\text { Gun- } \\
\text { licks } \\
\text { et al. } \\
2010\end{array}$ & $\begin{array}{l}\text { United } \\
\text { States }\end{array}$ & Depression & $\begin{array}{l}\text { Interpersonal } \\
\text { psychotherapy } \\
\text { for depressed } \\
\text { adolescents } \\
\text { week } 12 \\
\text { follow-up }\end{array}$ & $\begin{array}{l}\text { Interpersonal } \\
\text { Psychotherapy } \\
\text { group (IPT-A) } \\
\text { TG (n=31) } \\
\text { Treatment as } \\
\text { usual group } \\
\text { (TAU) } \\
\text { CG } \\
(\mathrm{n}=32) \\
\text { Age } m=15\end{array}$ & $\begin{array}{l}\text { Hamilton Rating } \\
\text { Scale for Depression } \\
\text { (HRSD) } \\
\text { Conflict Behavior } \\
\text { Questionnaire (CBQ- } \\
\text { 20) } \\
\text { CBQ_Mother } \\
\text { Social Adjustment } \\
\text { Scale - Self-report } \\
\text { (SAS-SR): }\end{array}$ & $\begin{array}{l}\text { (Assessed by Clinicians) } \\
\text { HRSD (at week 12) } \\
\text { TG } \downarrow \text { p }<.05 \\
\text { CBQ-20 } \leftrightarrow \\
\text { SAS-SR } \leftrightarrow\end{array}$ & $\begin{array}{l}\text { SAS-SR } \\
\text { Sub-scale: } \\
\text { Friends, } \\
\text { School,Famil } \\
\text { y, Dating } \\
\text { CBQ-20 }\end{array}$ \\
\hline
\end{tabular}


(Table 2) contd...

\begin{tabular}{|c|c|c|c|c|c|c|c|}
\hline Study & Country & Diagnosis & $\begin{array}{l}\text { Type of } \\
\text { program/FU }\end{array}$ & $\begin{array}{l}\text { Sample size } \\
\text { And group }\end{array}$ & Measures/outcome & Result & $\begin{array}{l}\text { Social } \\
\text { outcome }\end{array}$ \\
\hline $\begin{array}{l}\text { Rose, } \\
\text { et al. } \\
2014\end{array}$ & Australia & Depression & $\begin{array}{l}\text { Manualized } \\
\text { cognitive } \\
\text { behavior } \\
\text { Therapy and } \\
\text { Interpersonal } \\
\text { Psychotherapy } \\
\text { group program } \\
\text { (RAP) } \\
\text { Manualized } \\
\text { group Program } \\
\text { basic social } \\
\text { skills (PIR) } \\
\text { 12-month } \\
\text { follow-up }\end{array}$ & $\begin{array}{l}\text { CBT and } \\
\text { Interpersonal } \\
\text { Psychotherapy } \\
\text { (RAP) } \\
\text { TG1 (n=31) } \\
\text { Placebo, } \\
\text { exercises } \\
\text { therapeutically } \\
\text { inactive } \\
\text { CG } \\
(\mathrm{n}=31) \\
\text { Social skills } \\
\text { treatment group } \\
\text { TG2 (PIR) } \\
\text { (n=31) } \\
\text { Age m=13.5 }\end{array}$ & $\begin{array}{l}\text { Reynolds Adolescent } \\
\text { Depression Scale } \\
\text { Second Edition } \\
\text { (RADS-2). } \\
\text { Children's Depres- } \\
\text { sion Inventory (CDI) } \\
\text { Psychological Sense } \\
\text { of School Member- } \\
\text { ship (PSSM) } \\
\text { Clinical Assessment } \\
\text { of Interpersonal } \\
\text { Relations (CAIR) } \\
\text { Multidimensional } \\
\text { Students' Life } \\
\text { Satisfaction Scale } \\
\text { (MSLSS) } \\
\text { Clinician- } \\
\text { administered, semistr. } \\
\text { Interv.(DISCAP) }\end{array}$ & $\begin{array}{l}\text { (Assessed by Clinicians) } \\
\text { RADS-2 } \\
\leftrightarrow \text { (TG1) } \\
\text { TG2 } \downarrow \text { p }=.008 \text { (no at follow-up) } \\
\text { CDI } \\
\leftrightarrow \text { (TG1) } \\
\text { TG2 } \downarrow p=.026 \text { (not at follow-up) } \\
\text { PSSM } \\
\text { school connectedness } \\
\text { TG2 } \uparrow p=.061 \text { ) (not at follow-up) } \\
\leftrightarrow(\text { TG1) } \\
\text { But no difference on follow-up between TG1 and TG2 } \\
\text { MSLSS } \\
\text { TG2 } \uparrow p=.061 \\
\text { CAIR } \leftrightarrow \\
\text { DISCAP } \leftrightarrow\end{array}$ & $\begin{array}{l}\text { PSSM } \\
\text { CAIR } \\
\text { CDI: } \\
\text { Subscale } \\
\text { interpersonal } \\
\text { Problems }\end{array}$ \\
\hline $\begin{array}{l}\text { Tze- } \\
\text { Chun } \\
\text { Tang } \\
\text { et al. } \\
2009\end{array}$ & Taiwan & Depression & $\begin{array}{l}\text { Interpersonal } \\
\text { psychotherapy } \\
\text { Intervention } \\
\text { (IPT-A) } \\
\text { no follow-up }\end{array}$ & $\begin{array}{l}\text { Intensive } \\
\text { interpersonal } \\
\text { psychotherapy } \\
\text { TG (n=35) } \\
\text { Treatment as } \\
\text { usual (psycho- } \\
\text { education) } \\
\text { (TAU) CG } \\
(\mathrm{n}=328) \\
\text { Age } \mathrm{m}=15\end{array}$ & $\begin{array}{l}\text { Beck Anxiety Inven- } \\
\text { tory } \\
\text { (BAI) } \\
\text { Beck Depression } \\
\text { Inventory (BDI) } \\
\text { Beck Hopelessness } \\
\text { Scale (BHS) } \\
\text { Beck Scale for } \\
\text { Suicide } \\
\text { (BSS) }\end{array}$ & $\begin{array}{l}\text { (Assessed by Clinicians) } \\
\text { BAI } \\
\text { TG } \downarrow p<0.05 \\
\text { BDI } \\
\text { TG } \downarrow \text { p }<0.001 \\
\text { BHS } \\
\text { TG } \downarrow p<0.01 \\
\text { BSS } \\
\text { TG } \downarrow p<0.01\end{array}$ & \\
\hline $\begin{array}{l}\text { Stein, } \\
\text { et al. } \\
2003\end{array}$ & $\begin{array}{l}\text { United } \\
\text { States }\end{array}$ & $\begin{array}{l}\text { PTSD } \\
\text { post trau- } \\
\text { matic stress } \\
\text { disorders }\end{array}$ & $\begin{array}{l}\text { Child Cogni- } \\
\text { tive-behavioral } \\
\text { program }\end{array}$ & $\begin{array}{l}\text { Treatment } \\
\text { group TG } \\
(\mathrm{n}=61) \\
\text { Control } \\
\text { Group CG } \\
(\mathrm{n}=65) \\
(\text { Age } \mathrm{m}=11)\end{array}$ & $\begin{array}{l}\text { Child Ptsd Symptom } \\
\text { Scale (CPSS) } \\
\text { Child Depression } \\
\text { Inventory } \\
\text { (CDI) } \\
\text { Parents report Psy- } \\
\text { chosocial dysfunction } \\
\text { Teacher-Child Rating } \\
\text { Scale } \\
\text { (TCRS) }\end{array}$ & $\begin{array}{l}\text { (Assessed by Clinicians, Parents and Teachers) } \\
\text { CPSS } \\
\text { TG } \downarrow p<0.05 \\
\text { CDI } \\
\text { TG } \downarrow p<0.05 \\
\text { Parents report Psychosocial dysfunction } \\
\text { TG } \downarrow p<0.05 \\
\text { TCRS } \leftrightarrow\end{array}$ & $\begin{array}{l}\text { CPSS: } \\
\text { item relation- } \\
\text { ships with } \\
\text { friends and } \\
\text { item relation- } \\
\text { ships with } \\
\text { family } \\
\text { CDI: } \\
\text { Subscale } \\
\text { interpersonal } \\
\text { Problems } \\
\text { Parents report } \\
\text { Psychosocial } \\
\text { dysfunction }\end{array}$ \\
\hline $\begin{array}{l}\text { Tol WA, } \\
\text { et al. } \\
2010\end{array}$ & Sri Lanka & $\begin{array}{l}\text { PTSD } \\
\text { post trau- } \\
\text { matic stress } \\
\text { disorders }\end{array}$ & $\begin{array}{l}\text { Manualized } \\
\text { intervention of } \\
\text { cognitive } \\
\text { behavioral } \\
\text { Techniques and } \\
\text { creative ex- } \\
\text { pressive } \\
\text { elements } \\
\text { 3-month } \\
\text { follow-up }\end{array}$ & $\begin{array}{l}\text { Treatment } \\
\text { Group } \\
\text { TG }(\mathrm{n}=199) \\
\text { Waitlist group } \\
\text { CG } \\
(\mathrm{n}=200) \\
\text { Age } \mathrm{m}=11.03\end{array}$ & $\begin{array}{l}\text { Child PTSD Symp- } \\
\text { tom Scale (CPSS) } \\
\text { Depression Self- } \\
\text { Rating (DSRS) } \\
\text { Screen for Anxiety } \\
\text { Related Emotional } \\
\text { Disorders } \\
\text { (SCARED-5) }\end{array}$ & $\begin{array}{l}\text { (Assessed by Clinicians ) } \\
\text { PTSD } \leftrightarrow \\
\text { DSRS } \leftrightarrow \\
\text { SCARED-5 } \leftrightarrow\end{array}$ & $\begin{array}{l}\text { Secondary } \\
\text { outcome: } \\
\text { SDQ: } \\
\text { Prosocial } \\
\text { subscale }\end{array}$ \\
\hline
\end{tabular}


(Table 2) contd....

\begin{tabular}{|c|c|c|c|c|c|c|c|}
\hline Study & Country & Diagnosis & $\begin{array}{l}\text { Type of } \\
\text { program/FU }\end{array}$ & $\begin{array}{l}\text { Sample size } \\
\text { And group }\end{array}$ & Measures/outcome & Result & $\begin{array}{l}\text { Social } \\
\text { outcome }\end{array}$ \\
\hline $\begin{array}{l}\text { Kataoka } \\
\text { et al. } \\
2011\end{array}$ & $\begin{array}{l}\text { United } \\
\text { States }\end{array}$ & $\begin{array}{l}\text { PTSD } \\
\text { post trau- } \\
\text { matic stress } \\
\text { disorders }\end{array}$ & $\begin{array}{l}\text { Cognitive } \\
\text { behavioral } \\
\text { therapy skills } \\
\text { intervention in } \\
\text { a group } \\
\text { format (5-8 } \\
\text { students/group) }\end{array}$ & $\begin{array}{l}\text { Treatment } \\
\text { Group } \\
\text { TG }(n=61) \\
\text { Waitlist group } \\
\text { CG }(n=62) \\
\text { Age } m=11\end{array}$ & $\begin{array}{l}\text { Academic perform- } \\
\text { ance } \\
\text { (math and language } \\
\text { arts) } \\
\text { grades were extracted } \\
\text { from school records } \\
\text { and coded as } A=4 \text {, } \\
B=3, C=2, D=1 \text {, and } \\
F=0 \text { for use as an } \\
\text { outcome variable }\end{array}$ & $\begin{array}{l}\text { (Assessed by Teachers) } \\
\text { Math grade } \\
\text { TG } \uparrow \mathbf{p}=\mathbf{0 . 0 4 8} \text { ) } \\
\text { Language Arts } \leftrightarrow\end{array}$ & \\
\hline $\begin{array}{l}\text { Galla } \\
\text { et al. } \\
2011\end{array}$ & $\begin{array}{l}\text { United } \\
\text { States }\end{array}$ & Anxiety & $\begin{array}{l}\text { Modular } \\
\text { Cognitive } \\
\text { Behavioral } \\
\text { Therapy } \\
\text { Treatment } \\
\text { 1-year follow- } \\
\text { up }\end{array}$ & $\begin{array}{l}\text { Treatment } \\
\text { group TG } \\
(\mathrm{n}=14) \\
\text { Control group } \\
\text { CG }(\mathrm{n}=10) \\
\text { Age } \mathrm{m}=8.51 \text {, }\end{array}$ & $\begin{array}{l}\text { Child and Parent } \\
\text { Versions (ADIS- } \\
\text { C/P) } \\
\text { The Clinical Global } \\
\text { Impressions } \\
\text { Improvement scale } \\
\text { (CGI-I) } \\
\text { Multidimensional } \\
\text { Anxiety Scale } \\
\text { for Children } \\
\text { (MASC-C) }\end{array}$ & $\begin{array}{l}\text { (Assessed by Clinicians and Teachers) } \\
\text { Follow-up data have been only reported } \\
\text { TG ADIS-IV } \\
\text { TG } \downarrow p=.000 \\
\text { MASC-P } \\
\text { TG } \downarrow p=.006 \\
\text { MASC-C } \\
\text { TG } \downarrow p=.000 \\
\text { CGI } \leftrightarrow\end{array}$ & $\begin{array}{l}\text { MASC: } \\
\text { The Social } \\
\text { Anxiety scale } \\
\text { Humiliation/ } \\
\text { Rejection } \\
\text { subscale }\end{array}$ \\
\hline
\end{tabular}

$\leftrightarrow$ no statistical significance was found

$\uparrow$ a statistically significant increase was found

$\downarrow$ a statistically significant decrease was found

parents were suggestive of the effectiveness of these programs in reducing the symptoms of most mental disorders. Only two studies out of ten were not associated with improved outcomes in children. Three studies investigated training for teachers that specifically focused on improving the knowledge and management of school children's mental disease, and on decreasing attributional bias and stigma towards mental illness. The aim of these interventions was to improve school policies [21-23]. Effectiveness was assessed in terms of increased knowledge and more positive attitude towards talking about mental health (decrease of stigma) for both teachers and students. As depicted in Table 1, one study reported positive results; two interventions did not show improved outcomes in children $[22,23]$. The other seven studies did not set integration as their main goal. Two of these studies evaluated and confirmed the effectiveness of Cognitive Behavioral Therapy (CBT) with the participation of parents in a clinical sample of children with anxiety disorders. Both studies used clinical measures as outcome measures: Clinical Global Impressions (CGI), and Spence Children's Anxiety Scale (SCAS) [24, 25]. Two studies investigated the positive results of two combined manualized programs: Basic Incredible Years Parenting Programme and Dinosaur School Programme for children with Conduct Disorders (ODD). The two mixed programs included: positive discipline strategies, effective strategies for coping with stress, and social skills. They shared The Wally Child Social Problem-Solving Detective Game (WALLY) as main outcome, as shown in Tables $\mathbf{1}$ and $\mathbf{3}$ [8]. Finally, three studies were heterogeneous on clinical sample type (ADHD, Anxiety, ID) and verified the effectiveness of school programs that combine different techniques: CBT, behavioural techniques, skills training, family systems interventions and psychoeducation: Barkley's programme; Social Effectiveness Ther- apy for Children (SET-C), Parents Plus Children's Programme. They all used the Strengths and Difficulties Questionnaire (SDQ) as main outcome measure, as shown in Tables 2 and 3 [26-28].

\section{Treatment Effectiveness of School-based Interventions on Clinical Samples Targeting Pupils Only}

The overall findings about school-based treatment on clinical samples targeting pupils only were suggestive of the effectiveness of these programs in reducing the symptoms of most mental disorders. Only three studies were not associated with improved outcomes, and none of these specified integration as their specific goal. Most of the retrieved studies concerned a school-based intervention on a clinical sample with a target on pupils only $(n=10)$, and consisted of a mixture of CBT techniques. Some studies were manualized and concerned verified interventions in a school setting. One study, in particular, used the Resourceful Adolescent Program (RAP) [29] (Rose et al., 2014) that incorporates CBT and Interpersonal Psychotherapy (IPT-A) principles, including stress management skills, cognitive restructuring, problem solving, and conflict resolution within families. However the results of this study revealed that adolescents completing RAP did not report significantly reduced depressive symptoms. The preponderance of studies based on CBT concerned samples of school children with Depression Disorder $(n=4)$, one included treatment of Anxiety and Depression Disorders and Behavioural problems, one focused on Anxiety Disorder only, one on Behavioural problems, and three studies targeted Post-Traumatic Stress Disorder (PTSD) symptoms [29-38]. One of them did not identify any major effect on primary outcomes [36]. 
Table 3. Social outcome as possible indicator of social inclusion.

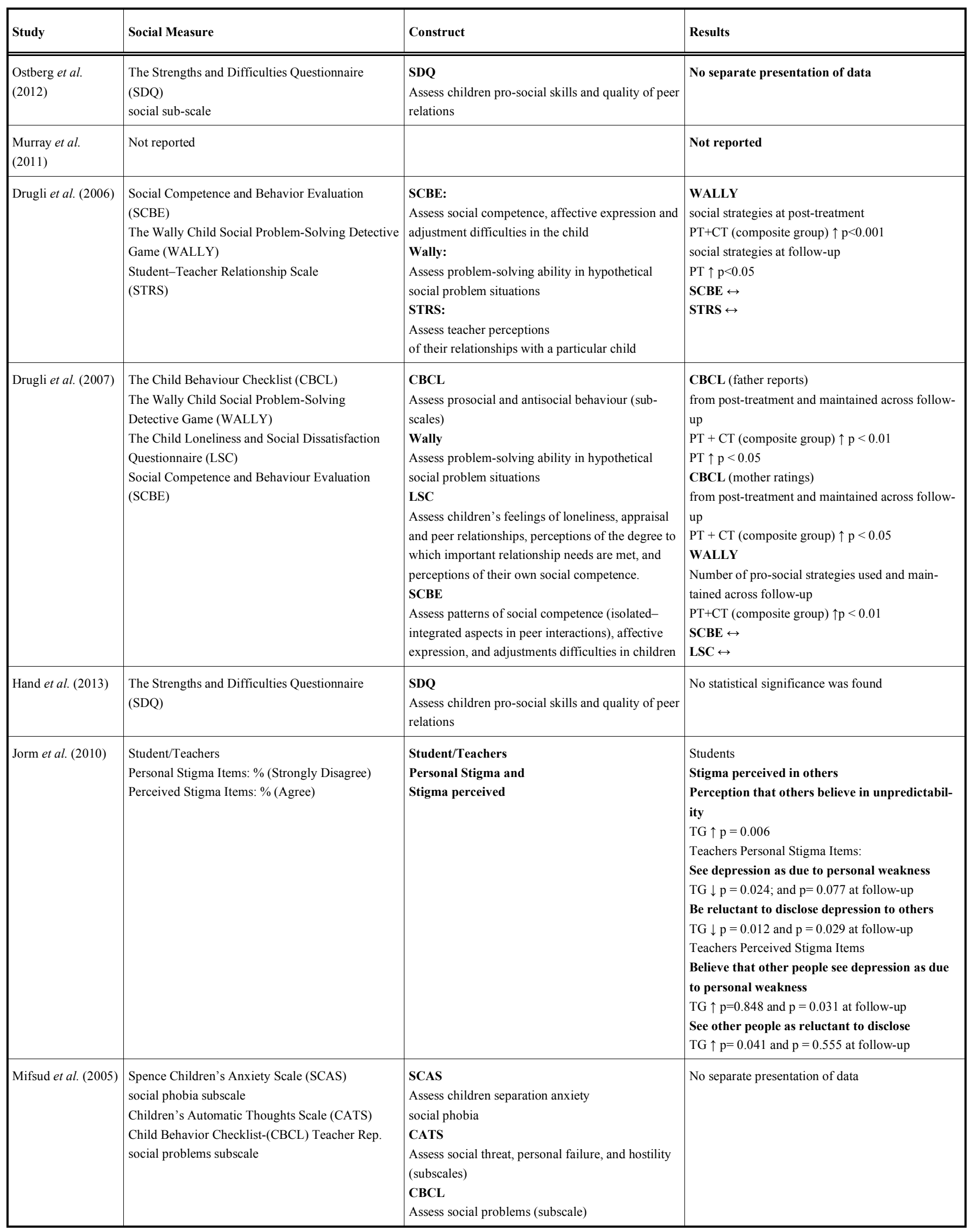


(Table 3) contd....

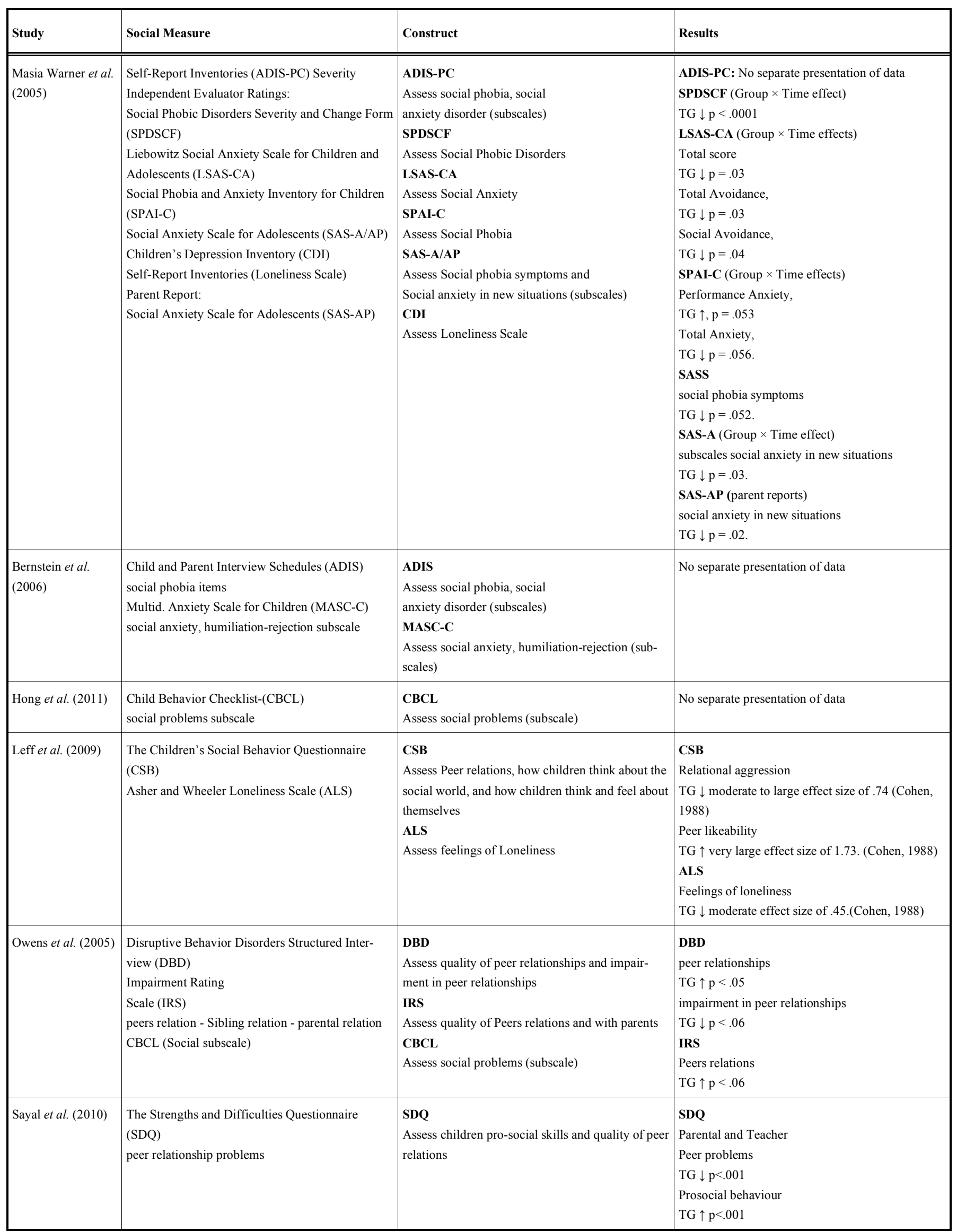


(Table 3) contd....

\begin{tabular}{|c|c|c|c|}
\hline Study & Social Measure & Construct & Results \\
\hline Cooper et al. (2010) & $\begin{array}{l}\text { The Strengths and Difficulties Questionnaire } \\
\text { (SDQ-PS) } \\
\text { prosocial subscale } \\
\text { Secondary outcome: } \\
\text { The Social Inclusion Questionnaire (SIQ) }\end{array}$ & $\begin{array}{l}\text { SDQ } \\
\text { Assess children pro-social skills and quality of peer } \\
\text { relations } \\
\text { SIQ } \\
\text { Assess Social Inclusion }\end{array}$ & $\begin{array}{l}\text { SDQ-PS } \\
\text { prosocial subscale } \\
\text { TG } \uparrow \mathrm{p}<.001\end{array}$ \\
\hline $\begin{array}{l}\text { Mufson et al. } \\
\text { (2004) }\end{array}$ & $\begin{array}{l}\text { Children's global assessment scale (C-GAS) } \\
\text { interaction with friends } \\
\text { Social adjustment scale-self report (SAS-SR) }\end{array}$ & $\begin{array}{l}\text { C-GAS } \\
\text { Assess quality of interaction with friends (sub- } \\
\text { scales) } \\
\text { SAS } \\
\text { Assess Social phobia symptoms and } \\
\text { Social anxiety in new situations (subscales) }\end{array}$ & $\begin{array}{l}\text { C-GAS } \\
\text { Social Functioning } \\
\text { dating } \\
\text { TG } \uparrow p=.03 \\
\text { overall social functioning } \\
\text { TG } \uparrow p=.01 \\
\text { family functioning } \\
\text { TG } \uparrow p=.10 \\
\text { SAS-SR. } \\
\text { TG } \uparrow p=.003\end{array}$ \\
\hline $\begin{array}{l}\text { O'Leary-Barrett } \\
\text { et al. }(2013)\end{array}$ & Not reported & & Not reported \\
\hline $\begin{array}{l}\text { Stallard et al. } \\
\text { (2012) }\end{array}$ & $\begin{array}{l}\text { Secondary outcomes: } \\
\text { Revised child anx. and dep. scale (RCADS) }\end{array}$ & $\begin{array}{l}\text { RCADS } \\
\text { Assess Social phobia } \\
\text { And quality of School connectedness } \\
\text { (subscales) }\end{array}$ & $\begin{array}{l}\text { RCADS } \\
\text { Social phobia } \\
\text { TG } \downarrow \mathrm{p}=.05 \\
\text { School connectedness } \\
\text { TG } \uparrow \mathrm{p}=.05\end{array}$ \\
\hline $\begin{array}{l}\text { Stallard et al. } \\
\text { (2013) }\end{array}$ & $\begin{array}{l}\text { Secondary outcomes: } \\
\text { Revised child anx. and dep. scale (RCADS) } \\
\text { Children's Automatic Thoughts Scale (CATS) } \\
\text { social phobia subscale }\end{array}$ & $\begin{array}{l}\text { RCADS } \\
\text { Assess Social phobia } \\
\text { And quality of School connectedness } \\
\text { (subscales) } \\
\text { CATS } \\
\text { Assess social threat, personal failure, and hostility } \\
\text { (subscales) }\end{array}$ & $\begin{array}{l}\text { RCADS } \\
\text { School connectedness } \\
\text { TG } \uparrow \mathrm{p}=.05 \\
\text { CATS } \\
\text { Social phobia sub-scale } \\
\text { TG } \downarrow \mathrm{p}=.05\end{array}$ \\
\hline $\begin{array}{l}\text { Manassis et al. } \\
(2010)\end{array}$ & $\begin{array}{l}\text { Multidimensional Anxiety Scale for Children } \\
\text { (MASC) social Anxiety scale, humiliation/rejection } \\
\text { subscale } \\
\text { Children's Depression Inventory (CDI) } \\
\text { Interpersonal Problems subscale }\end{array}$ & $\begin{array}{l}\text { MASC-C } \\
\text { Assess social anxiety, humiliation-rejection (sub- } \\
\text { scales) } \\
\text { CDI } \\
\text { Assess Loneliness Scale }\end{array}$ & No separate presentation of data \\
\hline $\begin{array}{l}\text { Gunlicks-Stoessel } \\
\text { et al. }(2010)\end{array}$ & $\begin{array}{l}\text { Social adjustment scale-self report } \\
\text { (SAS-SR) } \\
\text { Conflict Behavior Questionnaire (CBQ-20) }\end{array}$ & $\begin{array}{l}\text { SAS } \\
\text { Assess Social phobia symptoms and } \\
\text { Social anxiety in new situations (subscales) } \\
\text { CBQ } \\
\text { Assess levels of conflict in children 's relationship }\end{array}$ & No statistical significance was found \\
\hline Kirsten et al. (2014) & $\begin{array}{l}\text { Psychological Sense of School Membership } \\
\text { (PSSM) } \\
\text { CAIR } \\
\text { Children's Depression Inventory } \\
\text { (CDI) } \\
\text { Interpersonal Problems Subscale }\end{array}$ & $\begin{array}{l}\text { PSSM } \\
\text { Assess quality of psychological membership in } \\
\text { school } \\
\text { CAIR } \\
\text { Assess perceptions of children and adoles- } \\
\text { cents regarding the quality of their relationships } \\
\text { (Social, Family, and Academic) }\end{array}$ & $\begin{array}{l}\text { PSSM } \\
\text { School connectedness } \\
\mathrm{TG} \downarrow \mathrm{p}=.027 \\
\text { CAIR } \\
\text { Interpersonal peer relationships } \\
\text { TG } \uparrow \mathrm{p}=.010\end{array}$ \\
\hline $\begin{array}{l}\text { Tze-Chun Tanget } \\
\text { et al. (2009) }\end{array}$ & Not reported & & Not reported \\
\hline $\begin{array}{l}\text { Chemtob et al. } \\
\text { (2002) }\end{array}$ & Not reported & & Not reported \\
\hline Stein et al. (2003) & $\begin{array}{l}\text { Pediatric Symptom Checklist } \\
\text { Child Ptsd Symptom Scale (CPSS) } \\
\text { item relationships with friends and item relation- } \\
\text { ships with family } \\
\text { Children's Depression Inventory (CDI) } \\
\text { Interpersonal Problems subscale }\end{array}$ & $\begin{array}{l}\text { CPSS } \\
\text { Assess relationships with friends and item relation- } \\
\text { ships with family } \\
\text { CDI } \\
\text { Assess Loneliness Scale }\end{array}$ & $\begin{array}{l}\text { Pediatric Symptom Checklist } \\
\text { Psychosocial dysfunction } \\
\text { TG } \downarrow \text { p=.05 and maintain at 6-month }\end{array}$ \\
\hline
\end{tabular}


(Table 3) contd....

\begin{tabular}{|c|c|c|c|}
\hline Study & Social Measure & Construct & Results \\
\hline $\begin{array}{l}\text { Tol WA et al. } \\
\text { (2012) }\end{array}$ & $\begin{array}{l}\text { Secondary outcome: } \\
\text { The Strengths and Difficulties Questionnaire } \\
(S D Q) \\
\text { prosocial subscale }\end{array}$ & $\begin{array}{l}\text { SDQ } \\
\text { Assess children pro-social skills and quality of peer } \\
\text { relations }\end{array}$ & No statistical significance was found \\
\hline Brian et al. (2012) & $\begin{array}{l}\text { Multidimensional Anxiety Scale for Children } \\
\text { (MASC) } \\
\text { (The Social Anxiety scale Humiliation/Rejection } \\
\text { subscale) }\end{array}$ & $\begin{array}{l}\text { MASC-C } \\
\text { Assess social anxiety, humiliation-rejection (sub- } \\
\text { scales) }\end{array}$ & No separate presentation of data \\
\hline
\end{tabular}

$\leftrightarrow$ no statistical significance was found

$\uparrow$ a statistically significant increase was found

$\downarrow$ a statistically significant decrease was found

Three Interpersonal Psychotherapy (IPT-A) school-based interventions focused on Depression symptoms using Hamilton Rating Scale for Depression (HRSD) and Beck Depression Inventory (BDI) as main outcome. They reported significantly higher effects on reducing the severity of depression symptoms [39-41]. One intervention was performed as culturally-adapted social problem solving Intervention for Conduct problems (CD) in girls with relational aggression (GRAs) style. Greater decrease in teachers' reports about relational aggression from pre-treatment to post-treatment was found for students under treatment than for students in the control group. The main outcome measure in this study was derived from Asher and Wheeler Loneliness Scale Children's Depression Inventory [42]. One Behavioral Treatment intervention was performed on Attention deficit hyperactivity disorder (ADHD), Oppositional defiant disorder (ODD), Conduct problems (CD) and Disruptive Behavior Disorders (DBD) symptoms in a sample of schoolchildren. The study used the Disruptive Behavior Disorders Structured Interview (DBD) as main outcome measure and showed significant improvement [43]. Another study describing a school-based humanistic counselling intervention focused on the presence of Emotional distress symptoms in a sample of schoolchildren using The Self-Report Strengths and Difficulties Questionnaire (SDQ), but showed non-significant results on the primary outcome measure [44].

Finally one study reported results from a specific psychosocial treatment for children who showed symptoms of Post Traumatic Stress Disorder (PTSD) after Hurricane exposure. Data showed significant differences on the Kauai Recovery Inventory (KRI) after treatment and at follow-up (X months) [45] in treatment-group children, as compared to children in the control group.

\section{Social Outcome and Effectiveness of School-based Inter- ventions on Clinical Samples}

As stated above, only a few studies had a specific focus on investigating the integration of students with psychiatric diagnosis in the classroom; therefore all the studies that involved a clinical population in a school setting were included and changes in social outcome as a main or secondary outcome measure were reviewed. Five studies out of twenty- seven did not report a main outcome or secondary outcome measures as far as social inclusion or exclusion variables were concerned. It was not possible to extrapolate data from six studies, because for instance social variables were part of a subscale of the outcome measure that was used (are reported in Table 3 as No separate presentation of data).

Considering all the reviewed studies, the most frequently used measures of social variables were subscales of instruments such as the social subscale of The Strengths and Difficulties Questionnaire (SDQ), the Social Anxiety subscale and Humiliation/rejection subscale of the Multidimensional Anxiety Scale for Children (MASC), and the interpersonal problems subscale of the Children's Depression Inventory (CDI). The chosen instruments (though not always as the main outcome) that seemed to specifically focus on treatment effectiveness in relation to social dimensions were: Personal and Perceived Stigma Items, The Social Inclusion Questionnaire' (SIQ), Asher and Wheeler Loneliness Scale (ALS), the Psychological Sense of School Membership (PSSM), The Wally Child Social Problem-Solving Detective Game (WALLY) and the IRS (Peers relation, Sibling relation, Parental relation). Overall, as shown in Table 3, out of sixteen studies that reported indicators of social values or dimensions, thirteen studies reported positive results of social variables, while three studies found no effects on these outcomes.

\section{DISCUSSION}

This study assessed the literature on implemented and verified school-based practices addressed to clinical populations of students, and targeting specific mental disorders to improve the integration of pupils with specific mental health problems in the classroom and the school system. Of all the studies including clinical populations that were screened, only few concerned randomized controlled trials, which is the golden standard methodology to assess a program's effectiveness. In particular, this study evaluated the effectiveness of twenty-seven interventions on clinical populations. A larger amount of programs implementing standardized models with verifiable and evidence-based practices is still needed. Indeed in almost all the assessed studies, the main outcome, the effect size and the number required for treat- 
ment are not always clearly or fully indicated: it was therefore not possible to include these data in the tables. There is a clear call for the use of evidence-based practice (EBP) in schools. It is necessary to identify, better understand and define the potential barriers to the use of empirical interventions in school settings. This information could be used to guide strategies that promote EBP among school-based staff and clinicians [46]. A complicated issue in school interventions may involve the question on how all the elements of known evidence-based programs can fit in the complex network of a specific school community, and how these programs can be successfully and effectively implemented and coordinated [11]. Evidence-based practice has shown that through involvement in interventions, the whole school and its surrounding community as a unit of change produce better performance in promoting and reinforcing students' health behaviors [17, 18, 47]. Engaging teachers in proactive and cooperative classroom management may produce positive environments that encourage and reinforce health classroom behavior. School practices and interventions need to improve psychosocial functioning of school children in both school settings and at home, by involving parents, teachers, and pupils $[47,18,11,8]$ However, out of the twenty-seven studies included in this review only $37 \%$ involved the active participation of parents and teachers in school treatment. Therefore, the practices that proved to be the most effective are not always concretely implemented [11]. Very few studies have also involved parents as well as teachers and clinicians as evaluators in the assessment phase. Several evidences indicate significant differences between the different types of evaluation observers. This approach would make it possible to assess differences in effectiveness between different studies in a more reliable manner [48]. School-based treatments for mental disorders can also raise the risk of stigmatizing and over-diagnose students, and subsequently violate their social interactions and peer acceptance $[1,2]$. Social interactions can prevent the development of stigma towards mental disease, and young people's social networks are influential [49-51]. A lack of pro-social strategies is often disliked by peers and could result in social exclusion. For children with a mental disease, peer-rejection could exacerbate antisocial development, while acceptance by peers could buffer the effects of dysfunctional behaviours. For this reason interventions should also target peer acceptance and strengthen social competence in the school setting and in the community $[8,6,7,52]$.

To avoid the danger of stigmatization, there is some agreement that school-based programs should involve children in experiential activities, engage students' feelings and behaviour, and facilitate students' interaction with others. However, out of the twenty-seven studies included, only $59 \%$ considered social values or dimensions after treatment, $48 \%$ reported positive results on prosocial behaviors and quality of interactions as an outcome of the program, and maintained them at follow-up. More programs are needed that involve clinical populations of schoolchildren and implement standardized models of intervention, taking into account social inclusion outcome in the school setting. It would be necessary to monitor if these values and indicators of integration remain stable or change during the whole educational experience of students with mental disorders.

\section{CONFLICT OF INTEREST}

The authors confirm that this article content has no conflict of interest.

\section{ACKNOWLEDGEMENTS}

Declared none.

\section{REFERENCES}

[1] Schachter H, Girardi A, Ly M, et al. Effects of school-based interventions on mental health stigmatization: a systematic review. Child Adolesc Psychiatry Ment Health 2008; 2: 18.

[2] Angermeyer MC, Matschinger H. Social distance towards the mentally ill: results of representative surveys in the Federal Republic of Germany. Psychol Med 1997; 27(1): 131-41.

[3] Mura G, Carta MG, Helmich I, et al. Physical activity interventions in schools for improving lifestyle in European Countries. Clin Pract Epidemiol Mental Health 2015; 11: 77-101.

[4] Sancassiani F, Pintus E, Holte A, et al. Enhancing the emotional and social skills of the youth to promote their wellbeing and positive development: a systematic review of universal school-based randomized. Clin Pract Epidemiol Mental Health 2015; 11: 21-40.

[5] World Health Organization: Stigma and discrimination against the mentally ill in Europe. Briefing of the WHO European Ministerial Conference on Mental Health. Facing the Challenges, Building Solutions: 12-15 January 2005; Helsinki.

[6] Dodge KA, Lansford JE, Salzer BV, et al. Peer rejection and social information-processing factors in the development of aggressive behavior problems in children. Child Dev 2003; 74(2): 374-93.

[7] Webster-Stratton C, Lindsay DW. Social competence and conduct problems in young children: Issues in assessment. J Clin Child Psychol 1999; 28(1): 25-43.

[8] Drugli, MB, Larsson B. Children aged 4-8 years treated with parent training and child therapy because of conduct problems: generalization effects to day-care and school settings. Eur Child Adolesc Psychiatry $2007 ; 15(7): 392-9$.

[9] Webster-Stratton C, Lindsay DW (1999) Social competence and conduct problems in young children: Issues in assessment. J Clin Child Psychol 1999; 28(1): 25-43.

[10] Lowie JA, Lever NA, Ambrose MG, Tager SB, Hill S. Partnering with families in expanded school mental health programs. In: Weist MD, Evans SW, Lever NA, Eds. Handbook of School Mental Health: Advancing Practice and Research 2003: pp. 135-147.

[11] Greenberg MT, Weissberg RP, O'Brien MU, Zins JE, Fredericks L, Elias MJ. Enhancing school-based prevention and youth development through coordinated social, emotional, and academic learning. Am Psychol 2003; 58(6-7): 466-74.

[12] Sugai G, Horner RH. The evolution of discipline practices: Schoolwide positive behavior supports. Child Fam Behav Ther 2002; 24: 23-50.

[13] Putnam R, Handler M, Handler C, Ramirez P, Luiselli J. Improving student bus-riding behavior through a whole-school intervention. J Appl Behav Anal 2003; 36, 583-90.

[14] Nelson JR. Designing schools to meet the needs of students who exhibit disruptive behavior. J Emot Behav Disord 1996; 4: 147-61.

[15] Taylor-Greene S, Brown D, et al. Schoolwide behavior support: Starting the year off right. J Behav Edu 1997; 7: 99-112.

[16] Walker HM, Horner RH, Sugai G, et al. Inte grated approaches to preventing antisocial behavior patterns among school-age children and youth. J Emot Behav Disord 1996; 4:193-256.

[17] Durlak JA, Weissberg RP, Dymnicki AB, Schellinger KB. The impact of enhancing students' social and emotional learning: a meta-analysis of school-based universal interventions. Child Dev 2011; 82(1): 405-32.

[18] Weare K Nind M. Mental health promotion and problem prevention in schools: what does the evidence say? Health Promot Int 2011; 26 (suppl 1): i29-i69.

[19] Cantone E, Piras P, Vellante M, et al. Interventions on bullying and cyberbullying in schools: a systematic review. Clin Pract Epidemiol Mental Health 2015; 11: 58-76.

[20] Tajfel H, Billig MG, Bundy RP, Flament C. Social categorization and intergroup behavior. Eur J Soc Psychol 1971; 1(2): 149-78. 
[21] Murray DW, Rabiner DL, Hardy KK. Teacher management practices for first graders with attention problems. J Atten Disord 2011; 15(8): 638-45

[22] Jorm AF, Kitchener BA, Sawyer MG, Scales H, Cvetkovski S. Mental health first aid training for high school teachers: a cluster randomized trial. BMC Psychiatry 2010; 10: 51.

[23] Sayal K, Owen V, White K. Impact of early school-based screening and intervention programs for ADHD on children's outcomes and access to services: follow-up of a school-based trial at age 10 years. Arch Pediatric Adolescent Med 2010; 164(5): 462-9.

[24] Mifsud C, Rapee RM. Early intervention for childhood anxiety in a school setting: outcomes for an economically disadvantaged population. J Am Acad Child Adolesc Psychiatry 2005; 44(10), 9961004.

[25] Bernstein GA, Bernat DH, Victor AM, Layne AE. School-based interventions for anxious children: 3-, 6-, and 12-month follow-ups. J Am Acad Child Adolesc Psychiatry 2008; 47(9); 1039-47.

[26] Ostberg M, Rydell AM. An efficacy study of a combined parent and teacher management training program for children with ADHD. Nord J Psychiatry 2012; 66(2): 123-30.

[27] Masia-Warner C, Klein R, Dent H, et al. School-based intervention for adolescents with social anxiety disorder: results of a controlled study. J Abnorm Child Psychol 2005; 6: 707-22.

[28] Hand A, Raghallaigh CN, Cuppage J. A controlled clinical evaluation of the Parents Plus Children's Program for parents of children aged 6-12 with mild intellectual disability in a school setting. Clin Child Psychol Psychiatry 2013; 18(4): 536-55.

[29] Rose K, Hawes D, Hunt C. Randomized controlled trial of a friendship skills intervention on adolescent depressive symptoms. J Consult Clin Psychol 2014; 82(3): 510-20.

[30] Hong L, Yufeng W, Agh K. Preventing behavior problems among elementary schoolchildren: impact of a universal school-based program in China. J Sch Health 2011; 81(5): 273-80.

[31] O'Leary-Barrett M, Topper L, Al-Khudhairy N, et al. Two-year impact of personality-targeted, teacher-delivered interventions on youth internalizing and externalizing problems: a clusterrandomized trial. J Am Acad Child Adolesc Psychiatry 2013; 52(9): 911-20.

[32] Stallard P, Phillips R, Montgomery AA, et al. A cluster randomized controlled trial to determine the clinical effectiveness and costeffectiveness of classroom-based cognitive-behavioral therapy (CBT) in reducing symptoms of depression in high-risk adolescents. Health Technol Assess 2013; 17(47): vii-xvii, 1-109.

[33] Stallard P, Sayal K, Phillips R, et al. Classroom based cognitive behavioral therapy in reducing symptoms of depression in high risk adolescents: pragmatic cluster randomized controlled trial. BMJ 2012; 345: e6058.

[34] Manassis K, Wilansky-Traynor P, Farzan N, Kleiman V, Parker $\mathrm{K}$, Sanford M. The feelings club: randomized controlled evaluation of school-based CBT for anxious or depressive symptoms. Depress Anxiety 2010; 27(10): 945-52.

[35] Stein BD, Jaycox LH, Kataoka SH, et al. A mental health intervention for school children exposed to violence: a randomized controlled trial. J Am Med Assoc 2003; 290(5): 603-11.

[36] Tol WA, Komproe IH, Jordans MJ, et al. Outcomes and moderators of a preventive school-based mental health intervention for children affected by war in Sri Lanka: a cluster randomized trial. World Psychiatry 2012; 11(2): 114-22.

[37] Kataoka S, Jaycox LH, Wong M, et al. Effects on school outcomes in low-income minority youth: preliminary findings from a com- munity-partnered study of a school-based trauma intervention. Ethn Dis 2011; 21(3 Suppl 1): S1-71-7.

[38] Galla BM, Wood JJ, Chiu AW, et al. One year follow-up to modular cognitive behavioral therapy for the treatment of pediatric anxiety disorders in an elementary school setting. Child Psychiatry Hum Dev 2012; 43(2): 219-26.

[39] Mufson L, Dorta KP, Wickramaratne P, Nomura Y, Olfson $\mathrm{M}$, Weissman MM. A randomized effectiveness trial of interpersonal psychotherapy for depressed adolescents. J Consult Clin Psychol 2004; 82(3): 510-20.

[40] Gunlicks-Stoessel M, Mufson L, Jekal A, Turner JB. The impact of perceived interpersonal functioning on treatment for adolescent depression: IPT-A versus treatment as usual in school-based health clinics. J Consult Clin Psychol 2010; 78(2): 260-7.

[41] Tang TC, Jou SH, Ko CH, Huang SY, Yen CF. Randomized study of school-based intensive interpersonal psychotherapy for depressed adolescents with suicidal risk and par suicide behaviors. Psychiatry Clin Neurosci 2009; 63(4): 463-70.

[42] Leff SS, Gullan RL, Paskewich BS, et al. An initial evaluation of a culturally adapted social problem-solving and relational aggression prevention program for urban African-American relationally aggressive girls. J Prev Interv Community 2009; 37(4), 260-74.

[43] Owens JS, Richerson L, Beilstein EA, Crane A, Murphy CE, Vancouver JB. School based mental health programming for children with inattentive and disruptive behavior problems: first-year treatment outcome. J Atten Disord 2005; 9(1): 261-74.

[44] Cooper M, Rowland N, McArthur K, Pattison S, Cromarty K, Richards K. Randomized controlled trial of school-based humanistic counseling for emotional distress in young people: feasibility study and preliminary indications of efficacy. Child Adolesc Ment Health $2010 ; 22: 4-12$.

[45] Chemtob CM, Nakashima JP, Hamada RS. Psychosocial intervention for post disaster trauma symptoms in elementary school children: a controlled community field study. Arch Pediatr Adolesc Med 2002; 156(3): 211-6.

[46] Walker HM. Use of Evidence-Based Intervention in Schools: Where We've Been, Where We Are, and Where We Need to Go. School Psychol Rev 2004; 33(3): 398-407.

[47] Catalano R, Berglund ML, Ryan GAM, Lonczak HS, Hawkins JD. Positive youth development in the United States: Research findings on evaluations of positive youth development programs. Ann Am Acad Pol Soc Sci 2004; 591(1): 98-124.

[48] Sonuga-Barke EJ, Brandeis D, Cortese S, et al. Nonpharmacological interventions for ADHD: systematic review and meta-analyses of randomized controlled trials of dietary and psychological treatments. Am J Psychiatry 2013; 170(3): 275-89.

[49] Pinto-Foltz MD, Logsdon MC, Myers JA. Feasibility, acceptability, and initial efficacy of a knowledge-contact program to reduce mental illness stigma and improve mental health literacy in adolescents. Soc Sci Med 2011; 72(12): 2011-9.

[50] Pescosolido BA, Martin JK. Stigma and the sociological enterprise. In: Avison WR, Mcleod JD, Pescosolido BA, Eds. Mental Health, Social Mirror, NY: Springer 2007: pp. 307-28.

[51] Crosnoe R, McNeely C. Peer relations, adolescent behavior, and public health research and practice. Fam Community Health 2008 ; 31(15): S71-S80.

[52] Agabio R, Trincas G, Floris F, Carta MG. Sancassiani F, Angermeyer MC. A systematic review of school based alcohol and other drugs prevention programs. Clin Pract Epidemiol Mental Health 2015; 11: 102-112.

This is an open access article licensed under the terms of the Creative Commons Attribution Non-Commercial License (http:/creativecommons.org/licenses/by-nc/3.0/) which permits unrestricted, non-commercial use, distribution and reproduction in any medium, provided the work is properly cited 